\title{
ESTUDO COMPARATIVO DE SIMULAÇÕES PALEOCLIMÁTICAS NA REGIÃO DO ATLÂNTICO SUL
}

\author{
ADRIANA OLIVEIRA ${ }^{1}$, GABRIEL CLAUZET $^{2}$ e ILANA WAINER ${ }^{2}$ \\ ${ }^{1,2}$ Instituto Oceanográfico da Universidade de São Paulo, Departamento de Oceanografia Física, \\ Praça do Oceanógrafico, 191, Cidade Universitária, São Paulo, SP, Brasil. \\ CEP 05508-900. E-mail: adrianab@usp.br.
}

Recebido Março 2005 - Aceito Abril 2006

\begin{abstract}
RESUMO
Neste estudo são comparadas diferentes simulações de modelos paleoclimáticos para duas épocas distintas, o Último Máximo Glacial (UMG) e o Presente. Esta comparação visa elucidar as principais diferenças nos processos físicos e dinâmicos do sistema oceano-atmosfera na região do oceano Atlântico Sul entre estes dois períodos. Foram analisadas as variações na climatologia anual entre estas épocas para os seguintes parâmetros: temperatura do ar (TA), precipitação (PPT) e pressão ao nível do mar (PNM). As simulações numéricas analisadas são do projeto PMIP (Paleoclimate Modelling Intercomparison Project) e do modelo numérico acoplado NCAR (National Center for Atmospheric Research) CCSM (Comunnity Climate System Model) versão 1.4 nos dois períodos de interesse. Os resultados mostram uma intensificação do presente em relação ao UMG em todas as variáveis analisadas. As diferentes simulações atmosféricas do PMIP apresentaram padrões consistentes com os apresentados no modelo NCAR CCSM, sendo observados baixos valores de EQM (Erro Quadrático Médio) para grande parte da região de estudo.
\end{abstract}

Palavras-chave: Paleoclima, Último Máximo Glacial, Atlântico Sul.

\begin{abstract}
COMPARISON OF PALEOCLIMATICS SIMULATIONS IN THE SOUTH ATLANTIC REGION.

The present work presents a comparison between the results of different paleoclimate simulations models for two periods, the Last Glacial Maximum (LGM) and the Present day. This comparison intents to elucidate the overall differences of the physical and dynamical processes from ocean-atmosphere system for these two climates. We analyzed the variations in the annual climatology between the LGM and Present for the following variables: air temperature, precipitation and sea level pressure. We used the results of the PMIP (Paleoclimate Modelling Intercomparison Project) and the numerical coupled model developed at NCAR (National Center for Atmospheric Research) CCSM (Comunnity Climate System Model) for the two periods of interest. The results show an intensification of the present in relation to the LGM in all variables. The different atmospheric simulations of the PMIP showed similar patterns of those of the NCAR CCSM, where we observed low values of RMS in most of the region.
\end{abstract}

Keywords: Paleoclimate, Last Glacial Maximum, South Atlantic.

\section{INTRODUÇÃO}

A paleoclimatologia cresceu muito durante o último século e tem sido foco importante para entender o passado climático da Terra de forma a prever o clima no futuro. Estimar futuras alterações climáticas e seus efeitos requer uma compreensão de toda a faixa de variabilidade climática da Terra e de como os sistemas interligados gelo, oceano, atmosfera, continentes e biosfera respondem às mudanças climáticas.

Vários indícios geológicos mostram que nos últimos milhões de anos o clima da Terra tem flutuado entre estados glaciais e não glaciais. O Último Máximo Glacial (UMG) tem sido foco de interesse de diversos cientistas por apresentar o mais completo registro de todas as eras glaciais. Um rico banco de 
dados cobrindo este período é proveniente do projeto CLIMAP (Climate/Long-range Investigation, Mapping and Prediction) (1976). Este projeto foi desenvolvido em 1971 com cientistas de diversas instituições com o objetivo de reconstruir através de dados "proxy" as condições superficiais da Terra no UMG.

O período de interesse desse trabalho é o UMG relativo ao presente. Convencionou-se que o período de 21000 anos atrás $(21 \mathrm{~K})$ representa globalmente este evento. De fato, na literatura, a maioria dos estudos envolvendo modelos gerais de circulação se referem ao clima de $21 \mathrm{~K}$ como representativo da época de Máximo Glacial por exemplo, Williams et al., 1974; Gates, 1976; Manabe e Hahn, 1977; Hansen e Takahashi, 1984; Rind e Peteet, 1985; Jouzel et al., 1989; Lautenschlager e Herterich, 1990; Miller e Russel, 1989.

A mais notável característica do UMG foi a formação de grandes placas de gelo sobre os continentes e os oceanos. As maiores mudanças ocorreram na cobertura de gelo do Hemisfério Norte onde a frente oceânica polar e enormes blocos de gelo migraram em direção ao Equador. Ainda no Atlântico Norte, placas de gelo chegaram a atingir latitudes próximas a $45^{\circ} \mathrm{N}$, cobrindo todo o oceano nas latitudes mais altas durante o inverno. Este quadro resultou em uma vasta região da superfície da Terra $\left(120^{\circ} \mathrm{W}\right.$ a $90^{\circ} \mathrm{E}$, ao norte de $\left.45^{\circ} \mathrm{N}\right)$ coberta por gelo ou tundra, alterando significativamente a temperatura de todo o planeta.

Crosta et al. (1998) usaram uma técnica moderna aplicada a diatomâceas na Antártica para reconstruir quantitativamente a extensão sazonal de gelo marinho no UMG. No inverno o limite da extensão máxima no setor do Atlântico e oeste da Índia no oceano Austral atingia cerca de $48^{\circ} \mathrm{S}$. No verão a extensão máxima no UMG é similar a de hoje, o que contradiz as extensões encontradas pelo projeto CLIMAP (1981). Este projeto e o trabalho de Cooke e Hays (1982) estimaram uma cobertura de gelo permanente chegando a $50^{\circ} \mathrm{S}$ nos setores Atlântico e Índico do oceano Austral.

Vários estudos numéricos foram realizados com o intuito de simular o UMG: Manabe e Hahn (1977), utilizaram um modelo de circulação geral da atmosfera para analisar a região tropical. Foram investigadas duas condições de fronteira utilizando dados do CLIMAP (1976) para o UMG e para os dias atuais. Os resultados obtidos das simulações mostram a região continental tropical muito mais árida no UMG que atualmente, devido a fortes ventos saindo dos continentes. Esta intensificação dos ventos soprando dos continentes para os oceanos é resultado da queda de temperatura atmosférica sobre os continentes em relação aos oceanos durante o UMG.

Hansen e Takahashi (1984), desenvolveram uma série de experimentos utilizando modelos de circulação global para determinar a contribuição relativa de diferentes condições de fronteira. Analisando a temperatura global durante o UMG obtiveram valores da ordem de $3.5^{\circ}$ a $4^{\circ} \mathrm{C}$ menores que a temperatura global atual. Esta diferença foi atribuída a alterações na composição da atmosfera (por exemplo, na concentração de gás carbônico e material particulado) e na cobertura de nuvens durante os períodos estudados.

Shin (2002) estudando a circulação do oceano Atlântico no UMG observou que a água Profunda do Atlântico Norte (APAN) e a água Antártica de Fundo (AAF) estavam mais frias e salinas no UMG. Ao passo que a APAN está mais salina que a AAF no presente, no UMG a AAF estava mais salina que a APAN. Isto ocorreu por causa da liberação de grande quantidade de sal devido ao aumento de gelo, na formação da AAF.

Clauzet et al. (2004) mostram que na subsuperfície (até $500 \mathrm{~m}$ ) ocorre uma intensificação do transporte de massa no UMG no sul do Atlântico Sul $\left(35^{\circ} \mathrm{S}\right.$ a $\left.25^{\circ} \mathrm{S}\right)$ e um enfraquecimento do transporte no norte $\left(25^{\circ} \mathrm{S}\right.$ ao Equador). Nas camadas intermediárias do oceano há uma intensificação do giro subtropical e uma mudança em direção ao norte da bifurcação da Corrente Sul Equatorial no UMG. Isto leva a uma intensificação para sul do transporte de massa pela Corrente do Brasil e um enfraquecimento do transporte para norte.

Neste trabalho procura-se entender as mudanças climáticas ocorridas no Atlântico Sul durante o Último Máximo Glacial, utilizando o modelo de circulação geral acoplado do NCAR e cinco (5) simulações do PMIP. O objetivo principal deste trabalho é o de Intercomparar os resultados do modelo NCAR CCSM $1.4 \mathrm{com}$ as simulações do PMIP. Este último tem como foco somente a componente atmosférica enquanto o CCSM é um modelo de alta complexibilidade de interação das 4 componentes do sistema climático.

\section{MATERIAIS E MÉTODOS}

A fim de entender a variabilidade climática na região do oceano Atlântico Sul no modelo acoplado do National Center for Atmospheric Research (NCAR), CCSM (Community Climate System Model) versão 1.4 comparou-se os seus resultados com as simulações dos modelos do projeto PMIP, nos períodos de UMG e presente. São analisadas as variações na climatologia anual entre o UMG e o Presente para os parâmetros de temperatura do ar (TA), precipitação (PPT) e pressão ao nível do mar (PNM). A região de estudo compreende o oceano Atlântico Sul e o continente Sul Americano, mais especificamente entre $20^{\circ} \mathrm{N}-70^{\circ} \mathrm{S}$ e $100^{\circ} \mathrm{W}-20^{\circ} \mathrm{E}$. O impacto das mudanças climáticas nessa região e a variabilidade a elas associadas durante períodos extremos não são bem conhecidos, uma vez que a maior parte dos estudos sobre o UMG são focados no Hemisfério Norte. Estudos comparativos entre vários modelos forçados da mesma maneira, permitem entender que parte do sinal climático é físico e o que pode ser dependente de configuração numérica. 


\subsection{O modelo numérico NCAR CCSM 1.4}

Utilizou-se a versão 1.4 do modelo acoplado CCSM para simulações climáticas em grandes escalas de tempo (Otto-Bliesner e Brady, 2001). O modelo numérico acoplado é composto por 4 componentes: atmosférica, oceânica, superfície terrestre e gelo-marinho (Boville e Gent, 1998). A componente atmosférica deste modelo é a versão 3.6 do Community Climate Model, com resolução T31 (aproximadamente $3.75^{\circ}$ em latitude e longitude) e 18 níveis na vertical (maiores detalhes em Kiehl et al., 1998; Hack et al., 1998). A componente oceânica deste modelo foi desenvolvida no Geophysical Fluid Dynamics Laboratory (Gent et al., 1998). A resolução espacial é de $3.6^{\circ}$ na longitude, com resolução variável na latitude de $1.2^{\circ}$ nos trópicos para $2.3^{\circ}$ nos pólos, e 25 níveis na vertical. O modelo dinâmico do gelo do mar é baseado nos trabalhos de Flato e Hibler, 1992 e Weatherly et al., 1998. O modelo de superfície terrestre fornece um tratamento dos processos terrestres levando em conta os diferentes tipos de vegetação (Bonan, 1998).

A simulação controle (referente ao presente $(0 \mathrm{~K})$ ) foi rodada por 150 anos usando níveis de gases estufa pré-industriais (280 ppmv para o $\mathrm{CO}_{2}, 800$ ppbv para o $\mathrm{CH}_{4}, 275$ ppbv para $\mathrm{N}_{2} \mathrm{O}$ e 0 para clorofluorcarbonos). O UMG é integrado por 100 anos após o acoplamento das componentes, iniciado com condições que foram produzidas usando a TSM do CLIMAP (1981). A simulação do UMG usa concentrações de $\mathrm{CO}_{2}$ de 200 ppm, concentrações de gases estufa prescritas por Raynaud et al. (1993), topografia de gelo prescrita por Peltier (1994) e os parâmetros orbitais referentes ao UMG (Berger, 1978) para determinar o fluxo de calor total. Em ambas as simulações são utilizados os últimos 90 anos para calcular a média climatológica com a finalidade de manter a consistência entre os dois períodos. Mais detalhes sobre a simulação do UMG são descritas em Shin et al. (2003).

\subsection{As Simulações do Paleoclimate Modelling Intercomparison Project (PMIP)}

O PMIP (Joussaume e Taylor, 2000) é um projeto internacional envolvendo membros de grandes grupos de modelagem climática mundial e endossado pelo World Climate Research Programme (WCRP) e o International Geosphere-Biosphere Programme (IGBP) cujo objetivo é a intercomparação e análise de diferentes modelos numéricos aplicados ao estudo do paleoclima. Numa fase inicial, a componente atmosférica dos modelos climáticos (Modelos de circulação geral atmosférica: AGCMs) foi avaliada para Último Máximo Glacial (UMG: 21000 anos atrás, 21K) o
Holoceno Médio (6000 anos atrás, 6K) e o presente (0K). As simulações do UMG são analisadas para entender a resposta climática à presença de grandes placas de gelo, oceanos frios e baixa concentração de gases estufa. A extensão e espessura das placas de gelo são descritas por Peltier (1994), a extensão do gelo marinho e a temperatura da superfície do oceano são descritas por CLIMAP (1981).

Dentre estas diversas simulações atmosféricas cinco delas foram escolhidas para comparação com o modelo acoplado NCAR CCSM 1.4 (Tabela 1). Essas simulações possuem temperatura de superfície do mar (TSM) computada que segundo Pinot et al. (1999) são mais consistentes na reconstrução da temperatura que as simulações com TSM prescritas: O modelo MRI2 (Meteorological Research Institute), o modelo CCM1 (Community Climate Model), o modelo GEN2 ou GENESIS2 (Global and Environmental and Ecological Simulation of Interactive Systems), o modelo CCC2 (Canadian Centre for Climate), o modelo UKMO (United Kingdom Meteorological Office).

Todas as simulações do PMIP são rodadas com as mesmas condições de fronteira (Joussaume e Taylor 1995). A insolação é especificada como no UMG $(21 \mathrm{~K})$, embora a diferença na insolação entre o presente e o UMG seja menor que $1 \mathrm{~W} / \mathrm{m}^{2}$ nos trópicos (Berger 1978), e a forçante de insolação não desempenhe um papel importante para explicar mudanças no clima tropical do UMG. A concentração de $\mathrm{CO}_{2}$ no UMG é preescrita, conforme medidas dos registros de gelo (Barnola et al. 1987; Raynaud et al. 1993), como 200ppm.

Nessas simulações de TSM computadas utiliza-se de um modelo atmosférico acoplado com um modelo da camada de mistura do oceano, o transporte de calor (TC) é preescrito e aplicado em alguns pontos do oceano no UMG (maiores detalhes em Bonfils et al. 1998). Existem dois métodos para prescrever o TC nas simulações do PMIP: (a) inferindo o TC e associando-o com os experimentos do presente com TSM preescritas (CCC2, MRI2 e UKMO) e (b) inferindo o TC de observações estimadas (diferença entre a medida de radiação por satélite e o transporte atmosférico calculado) (CCM1 e GEN2) (Pinot et al. , 1999). Como se utiliza o TC como condição de contorno, essas simulações são forçadas com grande variedade de TC em contraste as simulações com TSM prescritas. Além disso, modelos com similar TC (por exemplo, UKMO e MRI2) possuem diferentes respostas às condições de gelo.

O esquema de superfície terrestre nos modelos do PMIP e sua resolução (ver tabela 1) são diferentes. Alguns modelos utilizam esquemas explícitos assim como o LSX (GEN2: Thompson e Pollard 1997), outros usam um modelo simples de "bucket" (CCM1 ou CCC2). 
Tabela 1 - Características dos modelos do PMIP utilizados nesse estudo e referências. Fonte: Pinot et al. 1999.

\begin{tabular}{ccccc}
\hline Modelos & País & Resolução & $\begin{array}{c}\text { Número de anos na } \\
\text { climatologia 0K/21K }\end{array}$ & Referências \\
\hline CCC2 & Canada & T32 $\left(96^{*} 48\right)$ & $10 / 10$ & Mc Farlane et al. 1992 \\
CCM1 & EUA & R15 $\left(48^{*} 40\right)$ & $10 / 10$ & Covey an Thompson 1989 \\
GEN2 & EUA & T31 $(96 * 48)$ & $10 / 10$ & Thompson and Pollard 1997 \\
MRI2 & Japão & $72 * 46$ & $14 / 14$ & Kitoh et al. 1999 \\
UKMO & Reino Unido & $96 * 73$ & $20 / 20$ & Hewitt and Mitchell 1997 \\
\hline
\end{tabular}

\subsection{Metodologia}

Para melhor caracterizar as diferenças entre os vários campos simulados utilizou-se o "Erro Quadrático Médio" (EQM) como técnica estatística. Com essa técnica pode-se comparar um campo de dados com um outro e verificar o quanto se distinguem entre si. Valores próximos a zero significam grande compatibilidade e quanto maior forem esses valores menos parecidos são os campos. Para obter o EQM primeiro calcula-se a diferença entre o UMG e o presente para cada modelo. Posteriormente calcula-se a diferença (S) entre a simulação acoplada do NCAR (S1) e as simulações do PMIP (S2), conforme equacionado abaixo:

S1 = NCAR CCSM 1.4 (UMG) - NCAR CCSM 1.4 (Presente)

$\mathrm{S} 2=\mathrm{PMIP}(\mathrm{UMG})$ - PMIP (Presente)

$\mathrm{S}=\mathrm{S} 1-\mathrm{S} 2$

Utilizando essa diferença calcula-se a variância que é a soma dos quadrados do desvio da média dividido pelo número total de medidas $n$ :

$$
\sigma^{2}=(1 / \mathrm{n}) \sum_{\mathrm{i}}^{\mathrm{i}=12}\left(\mathrm{x}_{\mathrm{i}}-\overline{\mathrm{x}}\right)^{2}
$$

onde $\overline{\mathrm{x}}$ é a média anual; $\mathrm{n}$ é o total de medidas e $\mathrm{x}_{\mathrm{i}}$ é o mês climatológico. $\mathrm{O}$ valor de EQM será igual à raiz quadrada da variância.

\section{RESULTADOS}

A análise dos resultados climatológicos foi feita de três formas. Primeiramente se analisou médias zonais compreendendo toda longitude da região de estudo $\left(100^{\circ} \mathrm{W}-20^{\circ} \mathrm{E}\right)$ para os três parâmetros escolhidos. Estas médias compreendem todas as simulações analisadas, permitindo uma comparação direta entre elas, para o UMG, presente e diferença entre os dois períodos. Posteriormente se calcula a diferença entre as climatologias anuais do UMG e presente para as seis simulações numéricas analisadas. Por fim foi calculado o EQM entre as diferenças obtidas do modelo acoplado do NCAR com as diferenças obtidas pelas demais simulações.

\subsection{Temperatura do Ar na Superfície (TA)}

A figura 1 mostra a média zonal de temperatura do ar para as simulações analisadas no presente, no UMG e para a diferença (UMG menos presente). Para o presente (fig.1a) o modelo do NCAR (linha cheia) mostra um mínimo valor de temperatura (aproximadamente $-10^{\circ} \mathrm{C}$ ) em $70^{\circ} \mathrm{S}$. A temperatura aumenta à medida que se caminha para o equador chegando a um máximo de aproximadamente $25^{\circ} \mathrm{C}$ entre $10^{\circ} \mathrm{S}$ e $10^{\circ} \mathrm{N}$. As simulações do PMIP seguem o mesmo padrão da curva do NCAR CCSM 1.4, porém próximo a região equatorial todos os modelos mostram valores inferiores aos do NCAR CCSM 1.4. Para o UMG (fig. 1b) a curva da média do modelo do NCAR (linha cheia) é similar a curva do presente. O valor mínimo é próximo a $-23^{\circ} \mathrm{C}$ e o máximo em torno de $22^{\circ} \mathrm{C}$. A fig.1c mostra que as maiores diferenças entre as simulações estão nas altas latitudes. Em $70^{\circ} \mathrm{S}$, a maior diferença é de aproximadamente $-13^{\circ} \mathrm{C}$ e a menor a $-4^{\circ} \mathrm{C}$. Entre as latitudes de $20^{\circ} \mathrm{S}$ e $0^{\circ}$ os valores das diferenças apresentam pouca variação $\left(2^{\circ}-4^{\circ} \mathrm{C}\right)$. O presente se apresenta mais quente que o $\mathrm{UMG}$ em todas as latitudes.

Apesar de características comuns entre os experimentos analisados a distribuição espacial da TA revela diferenças consideráveis entre os dois períodos. A figura 2 mostra as diferenças das climatologias anuais para os dois períodos de estudo para todos os modelos listados na tabela 1 e o modelo NCAR CCSM 1.4. As áreas negativas representam um aquecimento do presente em relação á $21 \mathrm{~K}$. Para todos os modelos estudados o presente apresenta valores de TA mais altos do que no UMG. Nos modelos NCAR CCSM 1.4 (fig.2a) e CCM1 (fig.2c) as diferenças na TA são superiores a $12^{\circ} \mathrm{C}$ e elas podem ser encontradas ao sul de $60^{\circ} \mathrm{S}$ sobre o oceano. Nota-se que a estrutura espacial das diferenças é consideravelmente diferentes, em particular sobre o continente, nos modelos MRI2, CCC2 e UKMO. Sobre o continente Sul Americano a diferença de temperatura é bastante alta com valores de aproximadamente $4^{\circ} \mathrm{C}$ conforme é observado nos modelos CCC2 (fig.2e) e CCM1 (fig.2c). O modelo UKMO 


\section{Media Zonal da Temperatura do Ar}

a)

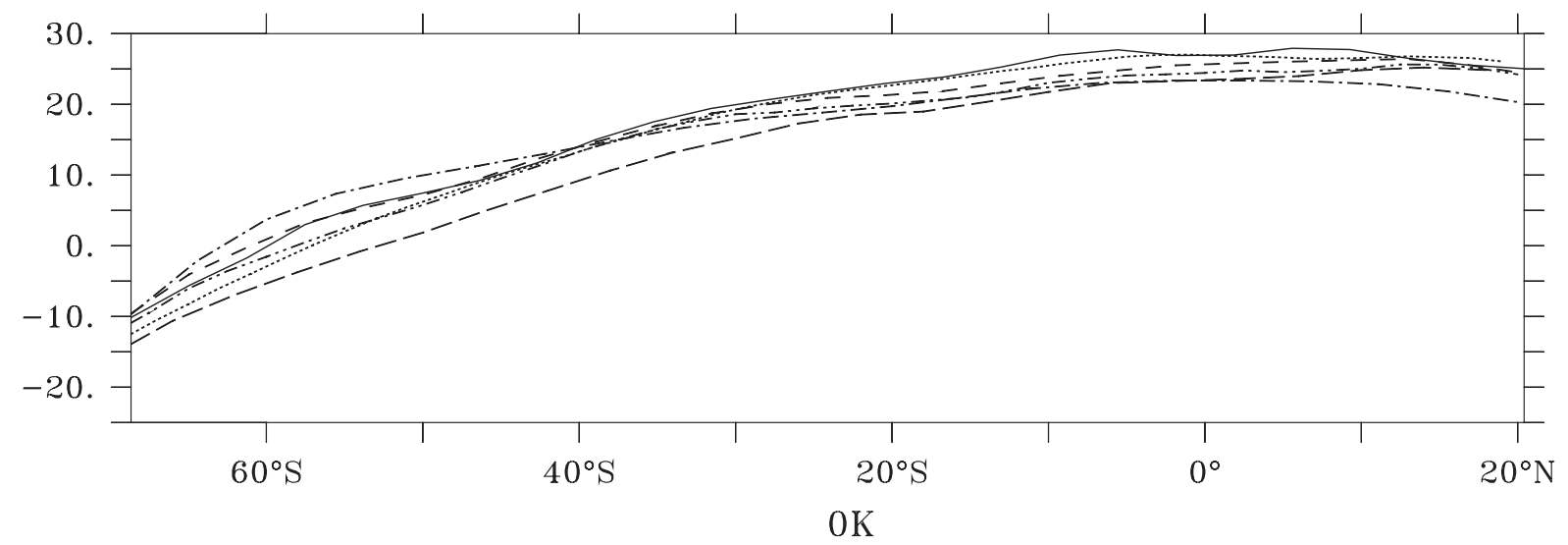

b)

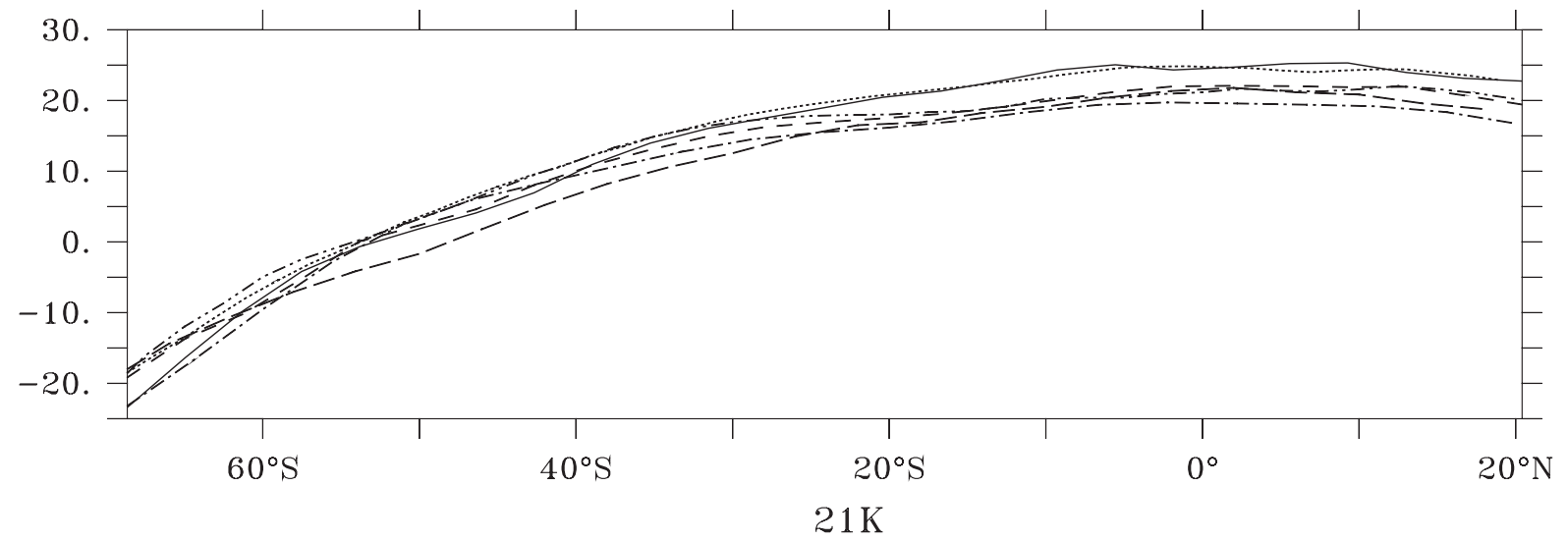

c)
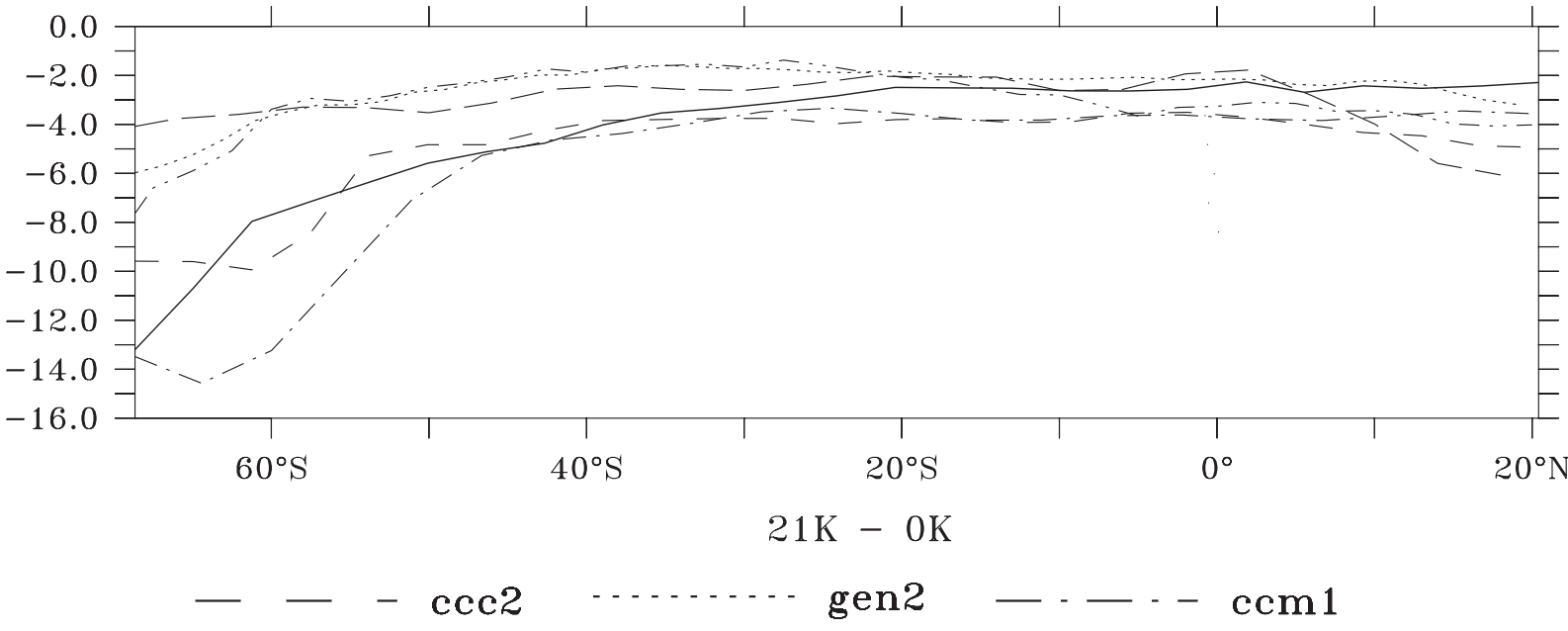

$--\operatorname{mri2} \quad \cdots+\cdots$ ukmo

Figura1 - Média zonal de temperatura do ar para as simulações analisadas no presente (a), no UMG (b) e para a diferença (UMG menos presente) (c). 
(fig.2f) apresenta diferenças sobre a região dos Andes (aproximadamente $2{ }^{\circ} \mathrm{C}$ ). Na região equatorial os modelos UKMO (fig.2f), GEN2 (fig.2d) e MRI2 (fig.2b) apresentam pequenas diferenças entre os dois períodos com valores em torno de zero, contrariamente o modelo MRI2 (fig.2b) também mostra valores de até $6{ }^{\circ} \mathrm{C}$.

A análise de EQM para a diferença entre o modelo NCAR CCSM 1.4 e os modelos do PMIP pode ser vista na Figura 3. Observa-se que os campos variam entre si muito pouco sendo a maior parte da região próxima a zero. Os modelos do PMIP apresentam maiores valores de EQM com relação ao modelo NCAR CCSM 1.4 principalmente ao sul de $40^{\circ} \mathrm{S}$ (figs. 3a-e). Nessas latitudes podemos observar valores de até $5^{\circ} \mathrm{C}$ de variação entre o NCAR CCSM 1.4 e o modelo CCM1.

\subsection{Precipitação (PPT)}

Considerando que a precipitação é fundamental para a determinação, por exemplo, do tipo de vegetação caracterizando o Paleoclima - A seguir se analisa sua representação nos modelos numéricos.

A figura 4 mostra a média zonal da precipitação para as simulações analisadas no presente, no UMG e para a diferença (UMG menos presente). As médias de precipitação mostram um padrão muito semelhante entre o UMG (fig.4b) e o presente (fig.4a), principalmente ao sul de $20^{\circ} \mathrm{S}$. Próximo a região equatorial as curvas dos modelos do PMIP apresentam maiores variações em relação ao modelo do NCAR para o presente (fig.4a) e UMG (fig.4b).

A diferença entre o presente e o UMG das climatologias anuais da PPT pode ser vista na fig.5. Em geral todas simulações mostram diferenças de um período para o outro podendo ser destacados valores ente -2 a $2 \mathrm{~mm} /$ dia de variação na maior parte da região de estudo. O oceano Atlântico Sul apresenta poucas diferenças na PPT entre os dois períodos em todos os modelos analisados. A região do modelo UKMO (fig.5f) situada entre 0 e $20^{\circ} \mathrm{S}$ de latitude e $10^{\circ} \mathrm{W}$ e $40^{\circ} \mathrm{W}$ de longitude apresenta maiores valores de precipitação no UMG do que no presente, esses valores chegam até a $4 \mathrm{~mm} /$ dia. A maior diferença entre todas as simulações está sobre o continente Sul-americano no modelo MRI2 (fig. 5b), com diferenças de $7 \mathrm{~mm} /$ dia.

A figura 6 apresenta os valores de EQM da precipitação entre o modelo NCAR CCSM 1.4 e as simulações do PMIP. Nesta figura pode-se observar que a maior parte da região de estudo, ao sul de $20^{\circ} \mathrm{S}$, apresenta valores próximos a 0 para todos os modelos indicando poucas diferenças. A região equatorial apresenta a maior variabilidade, principalmente sobre o continente da América do Sul, com núcleos de até 3mm/dia de EQM nos modelos UKMO (fig.6e), MRI2 (fig.6a) e GEN2 (fig.6c).

\subsection{Pressão Atmosférica ao Nível do Mar (PNM)}

A figura 7 mostra a média zonal de pressão atmosférica ao nível do mar para as simulações analisadas no presente (Fig.7a), no UMG (fig. 7b) e para a diferença (UMG menos presente) (fig.7c). Para o presente o modelo NCAR CCSM 1.4 possui um valor mínimo global em aproximadamente $65^{\circ} \mathrm{S}$ ( $\sim 985 \mathrm{mbar}$ ) e um valor máximo (1020mbar) em aproximadamente $35^{\circ} \mathrm{S}$. As curvas dos modelos do PMIP em baixas latitudes apresentam pequenas diferenças, enquanto as maiores se localizam nas altas latitudes. O modelo CCM1 difere bastante dos demais, não evidenciando a alta subtropical. Para o UMG o modelo NCAR CCSM 1.4 apresenta o mesmo padrão do presente, porém a alta subtropical é menos intensa ( 1015 mbar). Os modelos do PMIP apresentam maiores variações em relação ao modelo NCAR CCSM 1.4. A fig. $7 \mathrm{c}$ mostra maiores diferenças nas altas latitudes em torno de 3 mbar maior no presente para a simulação do NCAR. Os modelos UKMO e MRI2 apresentam as maiores diferenças, cerca de 6 e 11 mbar respectivamente em todas as latitudes, indicando uma maior pressão atmosférica no UMG que no presente. Essas diferenças são consistentes com os campos de temperatura e precipitação, ou seja, há manifestação de sensibilidade tanto nas altas latitudes quanto na região da Zona de Convergência Intertropical (ITCZ); particularmente para os modelos MRI2 e UKMO.

As climatologias anuais da PNM para as diferenças entre o UMG e presente dos modelos é apresentada na figura 8. Apesar da PNM estar diretamente relacionada às variações de temperatura, a diferença entre os períodos climáticos não é evidente no oceano Atlântico Sul. As maiores diferenças sobre toda a região de estudo estão no modelo MRI2 (fig.8b) onde os valores são da ordem de $15 \mathrm{mb}$ maiores no UMG que no presente, o que ocorre também no modelo UKMO (fig.8f) em menor magnitude, de acordo com o observado na fig.7c. Os modelos CCC2, CCM1, NCAR CCSM 1.4 e GEN2 (figs. 8e, 8c, $8 \mathrm{a}$ e $8 \mathrm{~d}$ ) apresentam valores na maior parte da região de estudo negativos, ou seja, ocorreu em média uma intensificação da PNM no presente em relação ao UMG, com valores próximos a $2 \mathrm{mb}$ de diferença.

Pode-se observar na figura 9 que a análise de EQM para a diferença entre o modelo NCAR CCSM 1.4 e os modelos do PMIP apresentam sobre toda região de estudo variabilidade de aproximadamente $2-3 \mathrm{mb}$. As regiões das altas latitudes foram as que apresentaram os maiores valores, os modelos CCC2, CCM1 e GEN2 (figs. 6d, 6b e 6c) apresentam valores de EQM próximos a $3 \mathrm{mb}$ 
Temperatura do $\mathrm{AR}-21 \mathrm{~K}-0 \mathrm{~K}$

a)

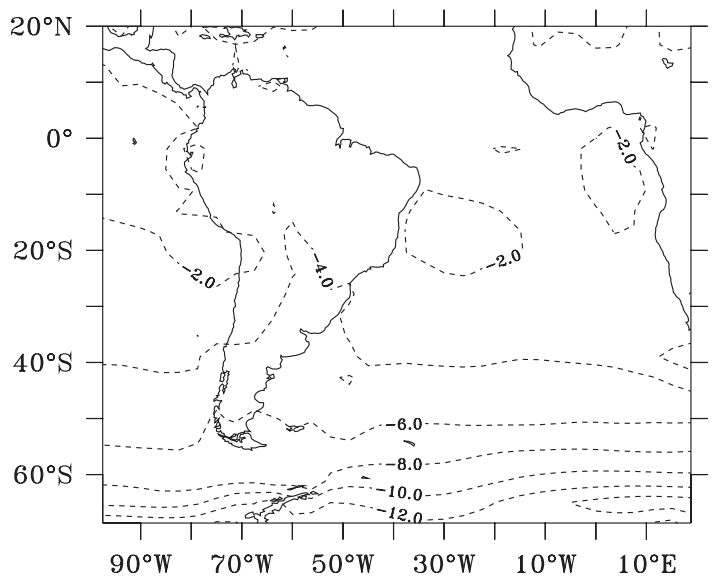

NCAR CCSM

c)

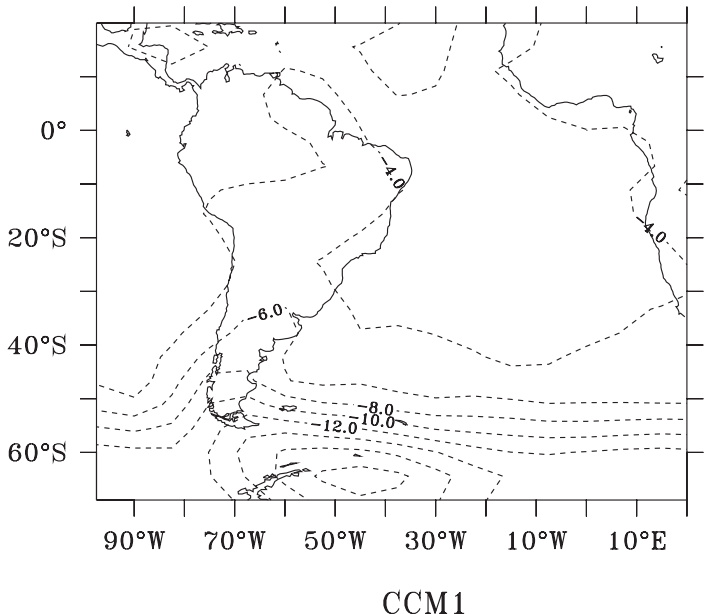

e)

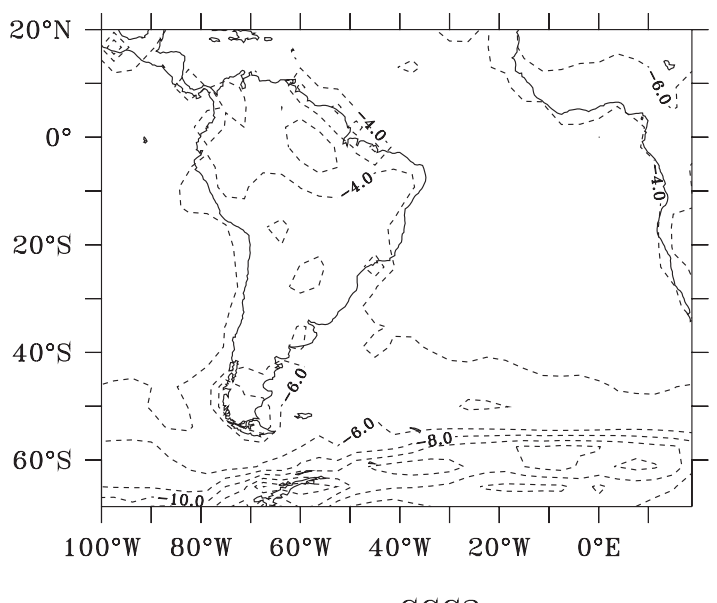

b)

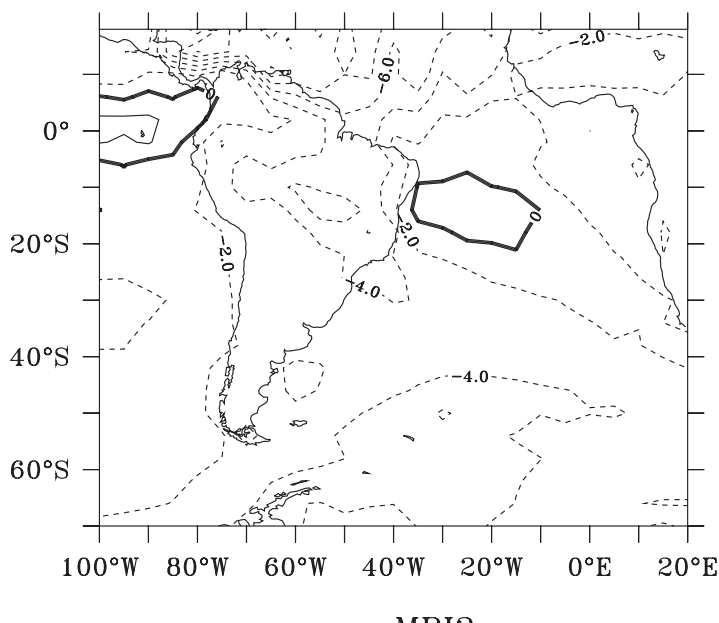

MRI2

d)

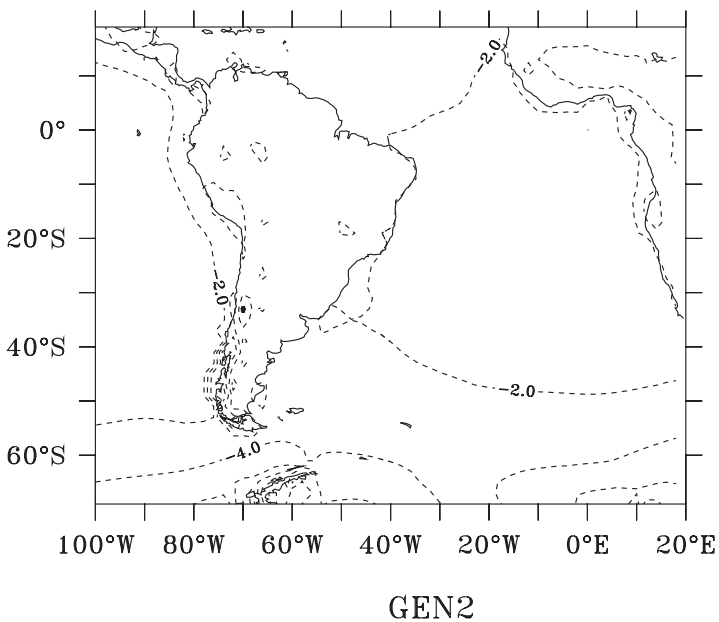

f)

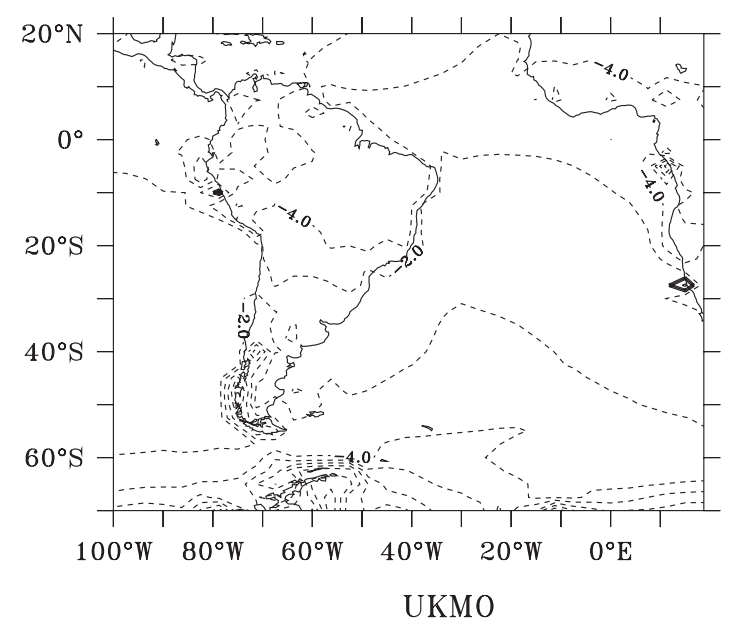

Figura 2 - Diferença das climatologias anuais da temperatura do ar entre o UMG e o presente dos modelos. Contornos de intervalos de $2^{\circ} \mathrm{C}$. (a) NCAR CCSM 1.4, (b) MRI2, (c) CCM1, (d) GEN2, (e) CCC2, (f) UKMO. 
Temperatura do AR - EQM

a)

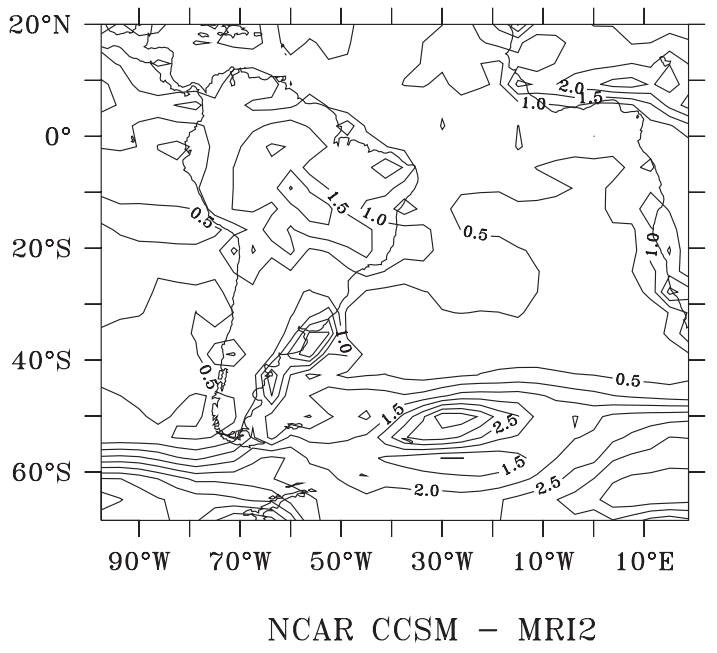

c)

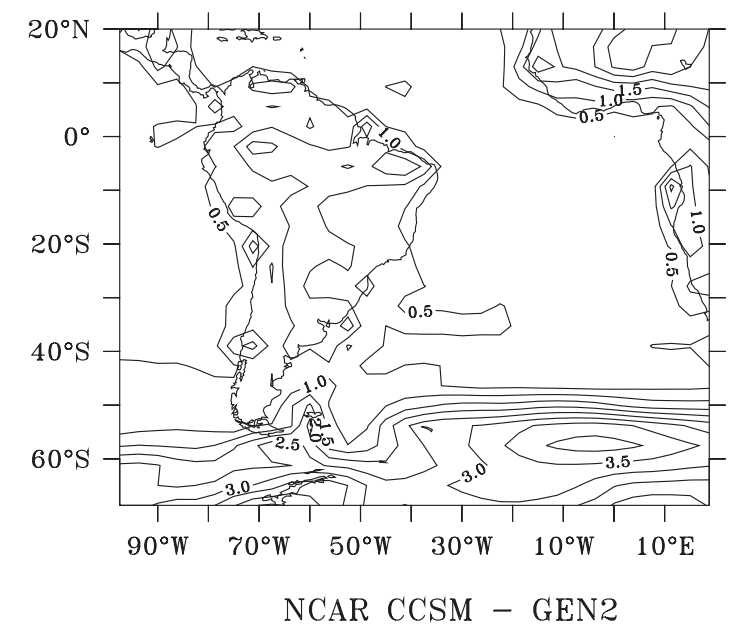

b)

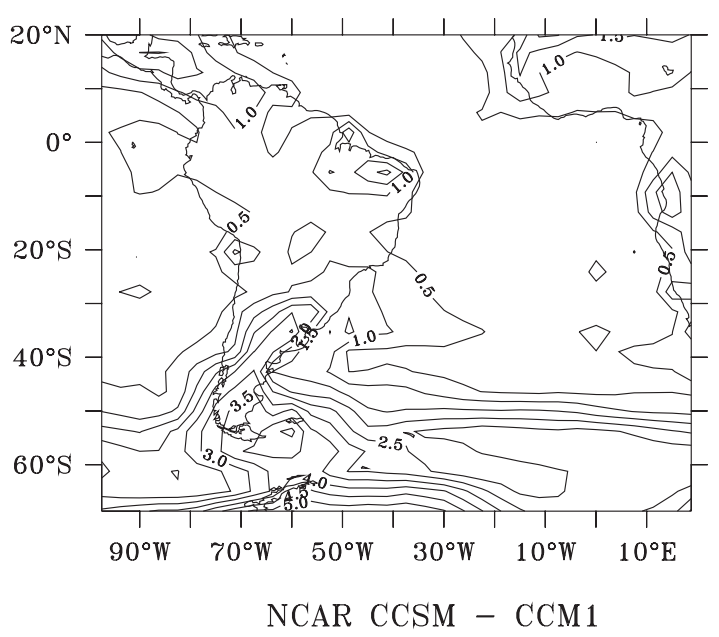

d)

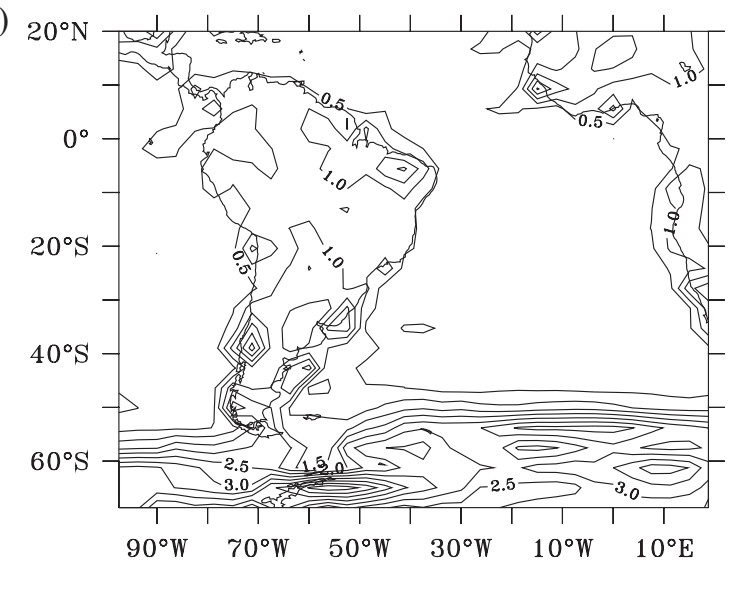

NCAR CCSM - CCC2

e)

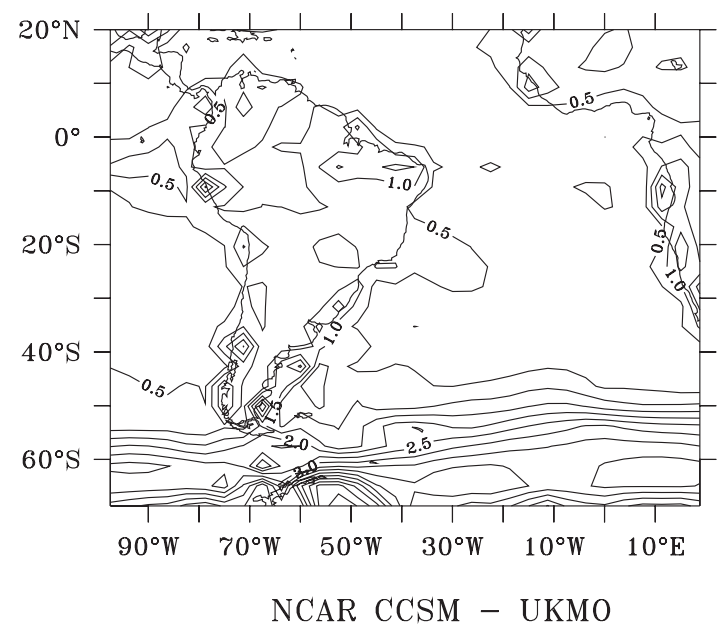

Figura 3 - Análise de EQM para a diferença S entre o modelo NCAR-CCSM 1.4 e os modelos do PMIP. Contornos de intervalos de $0.5^{\circ} \mathrm{C}$. (a) NCAR CCSM 1.4 - MRI2, (b) NCAR CCSM 1.4 - CCM1, (c) NCAR CCSM 1.4 - GEN2, (d) NCAR CCSM 1.4 - CCC2, (e) NCAR CCSM 1.4 - UKMO. 


\section{Media Zonal da Precipitacao}

a)

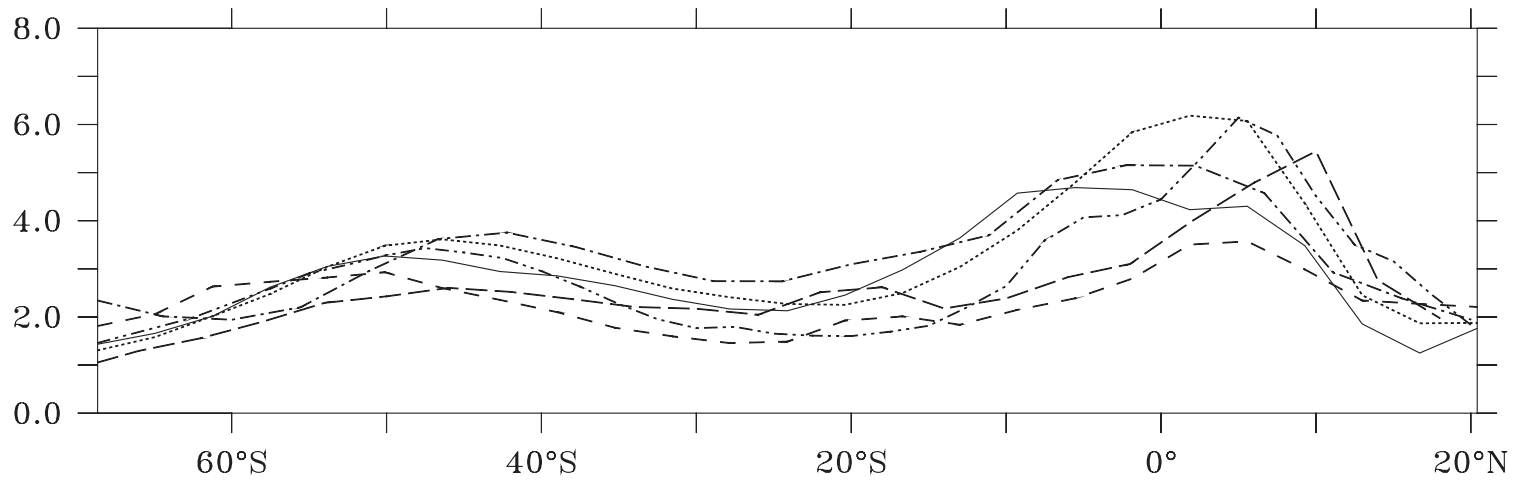

OK

b)

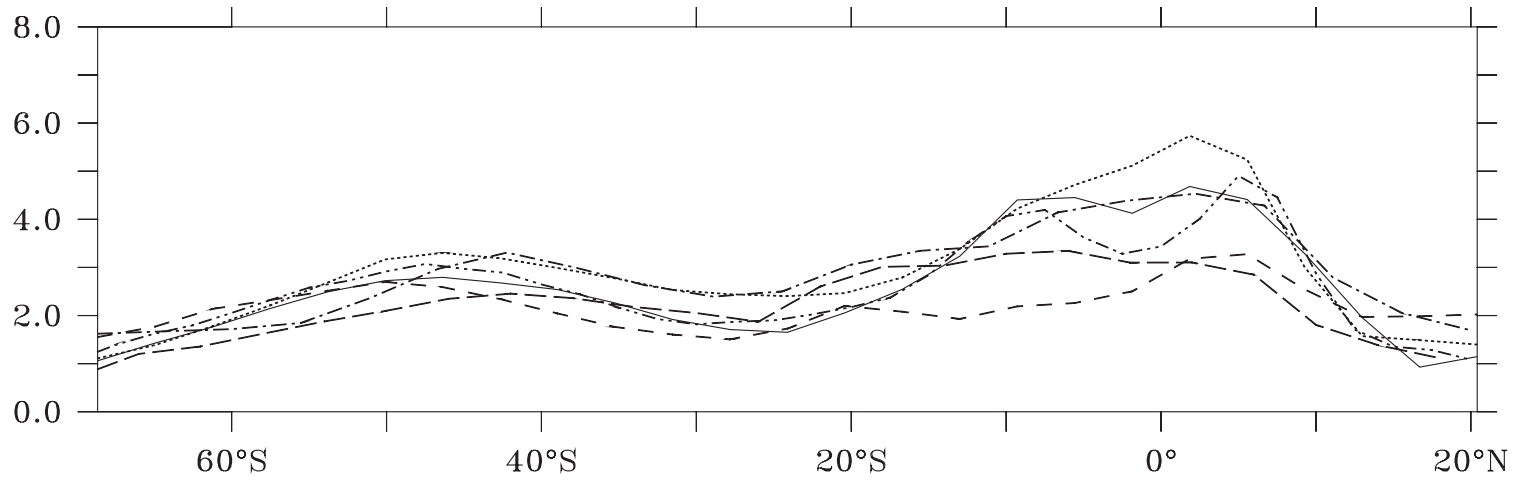

$21 \mathrm{~K}$

c)

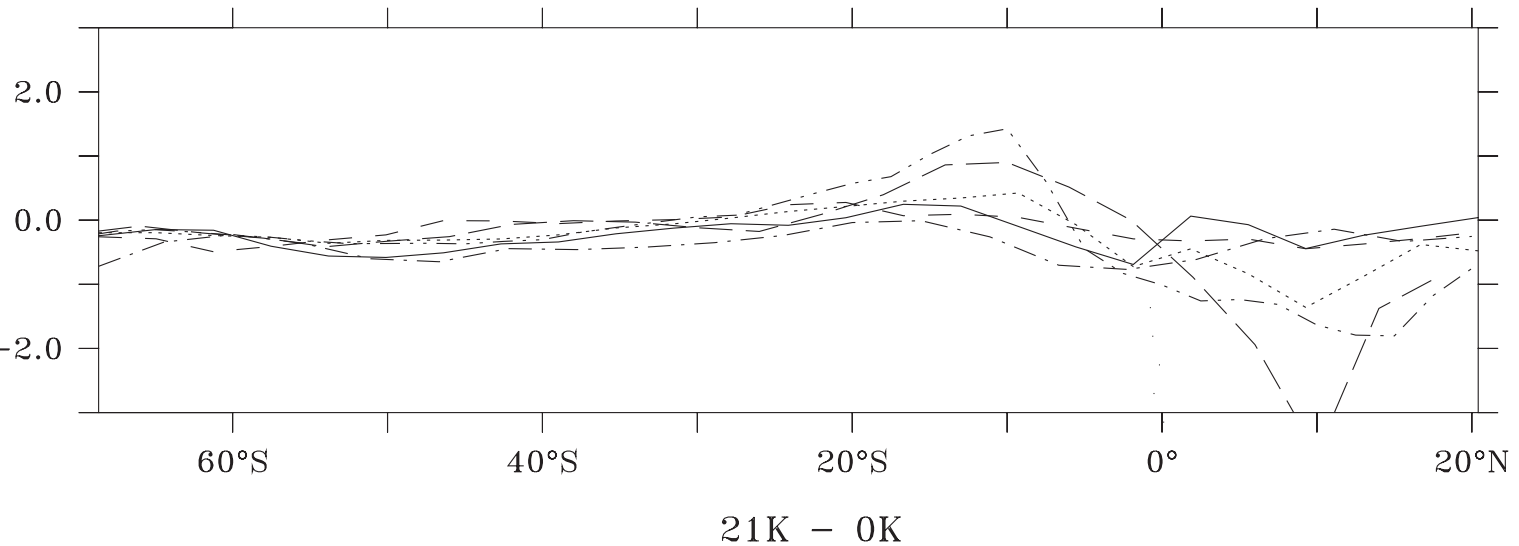

$-\quad-\operatorname{ccc} 2 \cdots \cdots \cdot \operatorname{gen} 2-\cdots-\operatorname{ccm} 1$

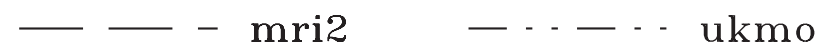

Figura 4 - Média zonal de precipitação para as simulações analisadas no presente (a), no UMG (b) e para a diferença (UMG menos presente) (c). 
Precipitacao - $21 \mathrm{~K}-0 \mathrm{~K}$

a)

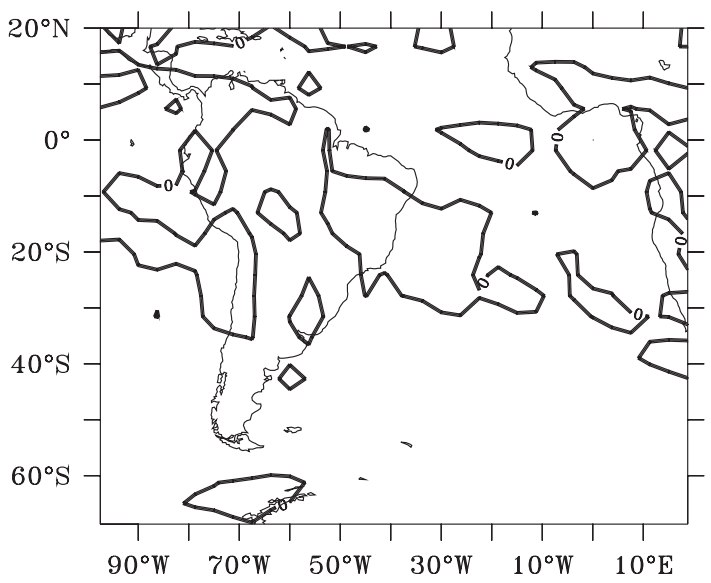

NCAR CCSM

c)

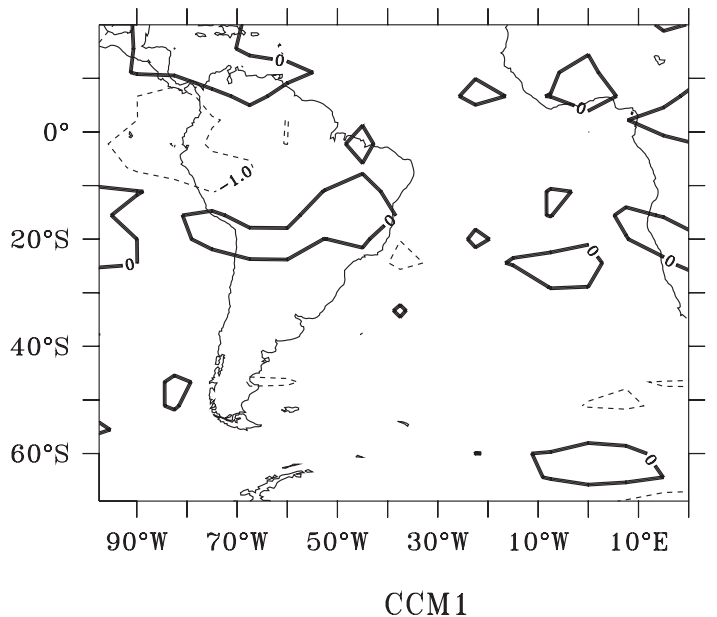

e)

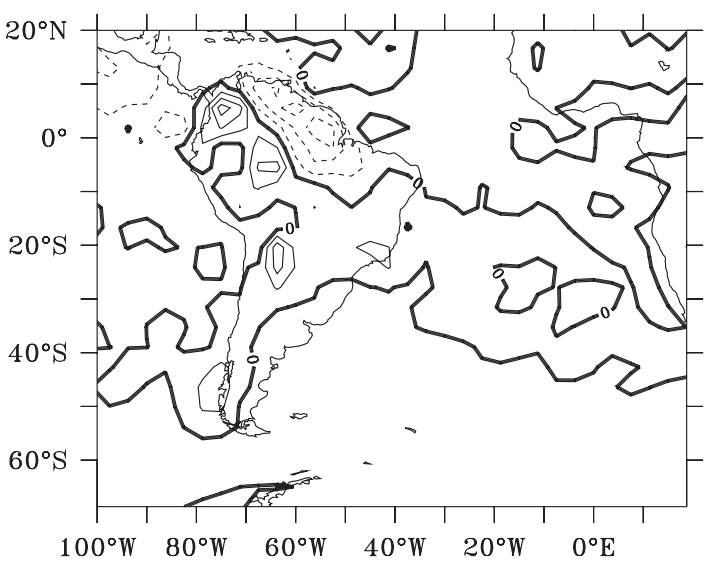

$\mathrm{CCC} 2$ b)

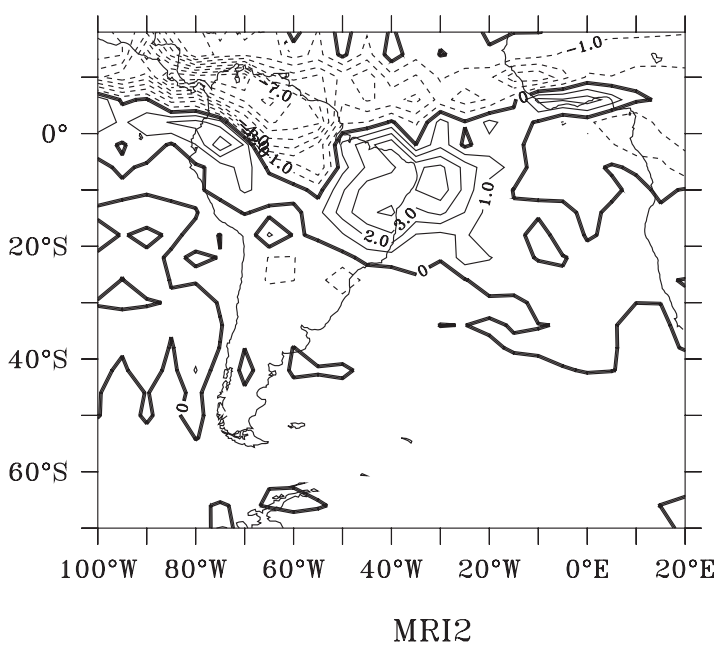

d)

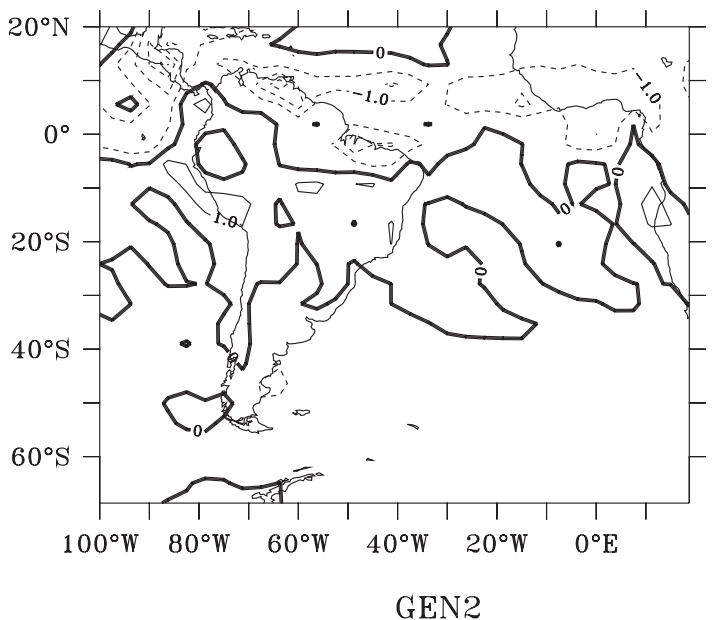

f)

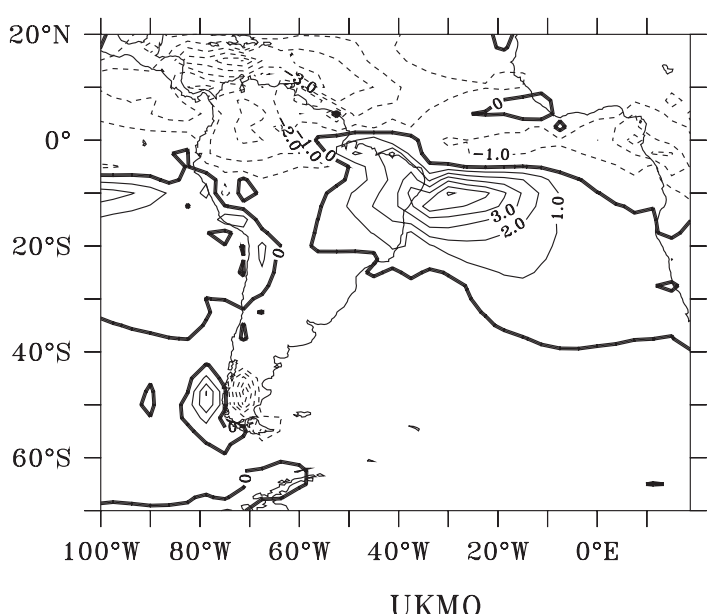

Figura 5 - Diferença das climatologias anuais da precipitação entre os dois períodos estudados dos modelos. Contornos de intervalos de 1mm/dia. (a) NCAR CCSM 1.4, (b) MRI2, (c) CCM1, (d) GEN2, (e) CCC2, (f) UKMO. 


\section{Precipitacao - EQM}

a)

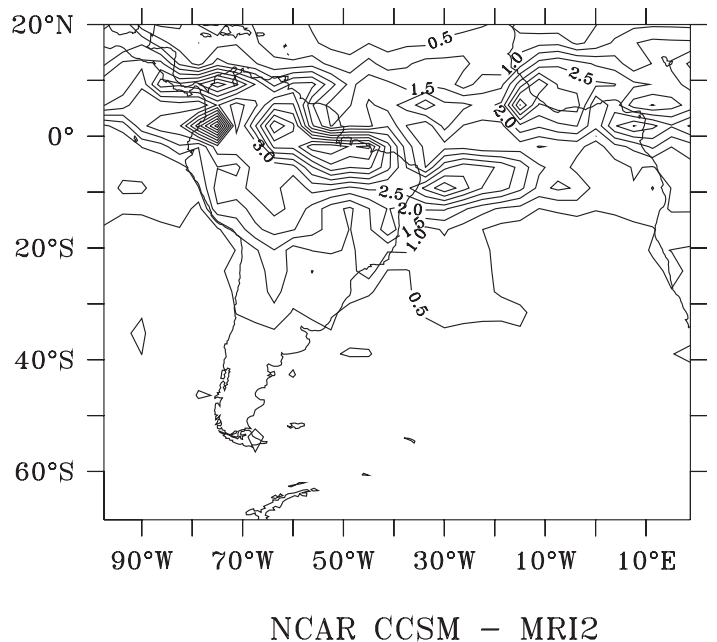

c)

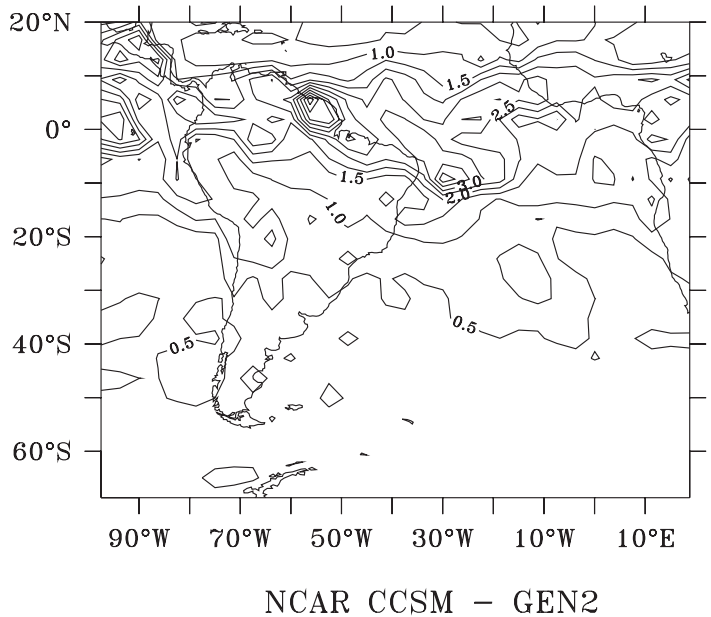

b)

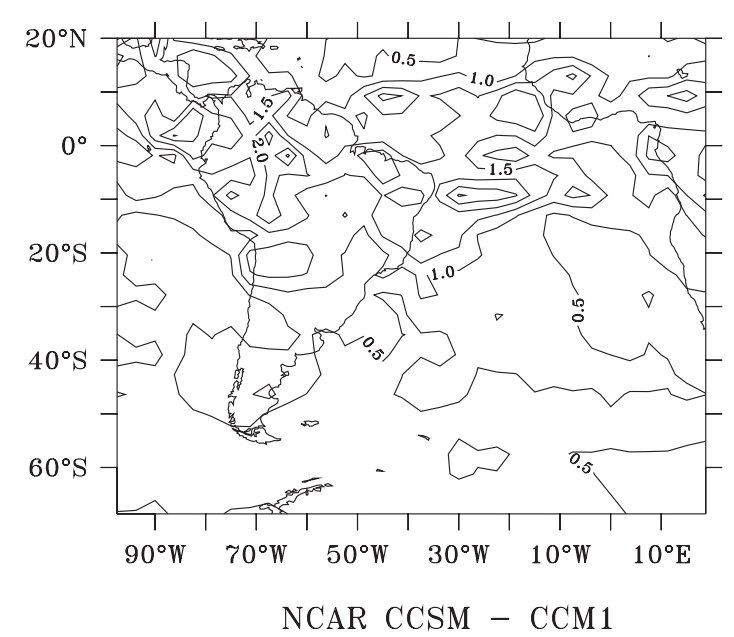

d)

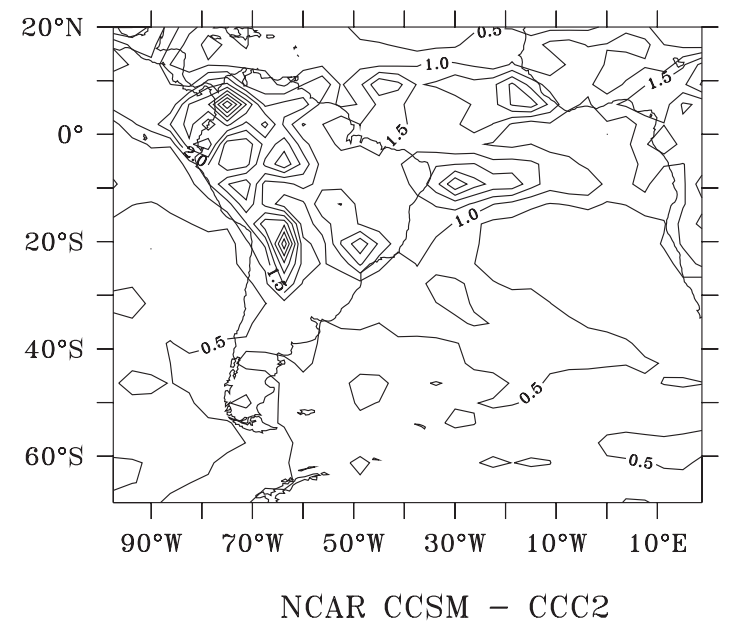

e)

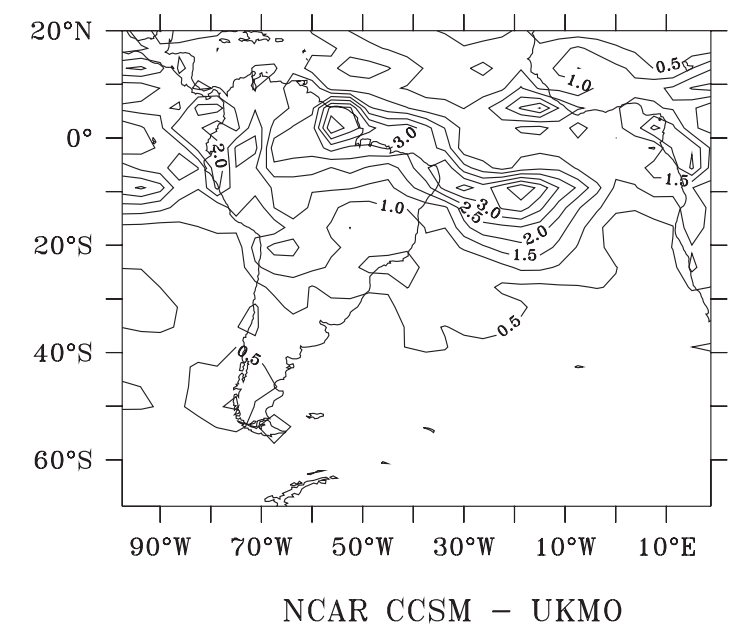

Figura 6 - Análise de EQM para a diferença S entre o modelo NCAR-CCSM 1.4 e os modelos do PMIP. Contornos de intervalos de $0.5 \mathrm{~mm} /$ dia. (a) NCAR CCSM 1.4 - MRI2, (b) NCAR CCSM 1.4 - CCM1, (c) NCAR CCSM 1.4 - GEN2, (d) NCAR CCSM 1.4 - CCC2, (e) NCAR CCSM 1.4 - UKMO. 


\section{Media Zonal da Pressao ao Nivel do Mar}

a)

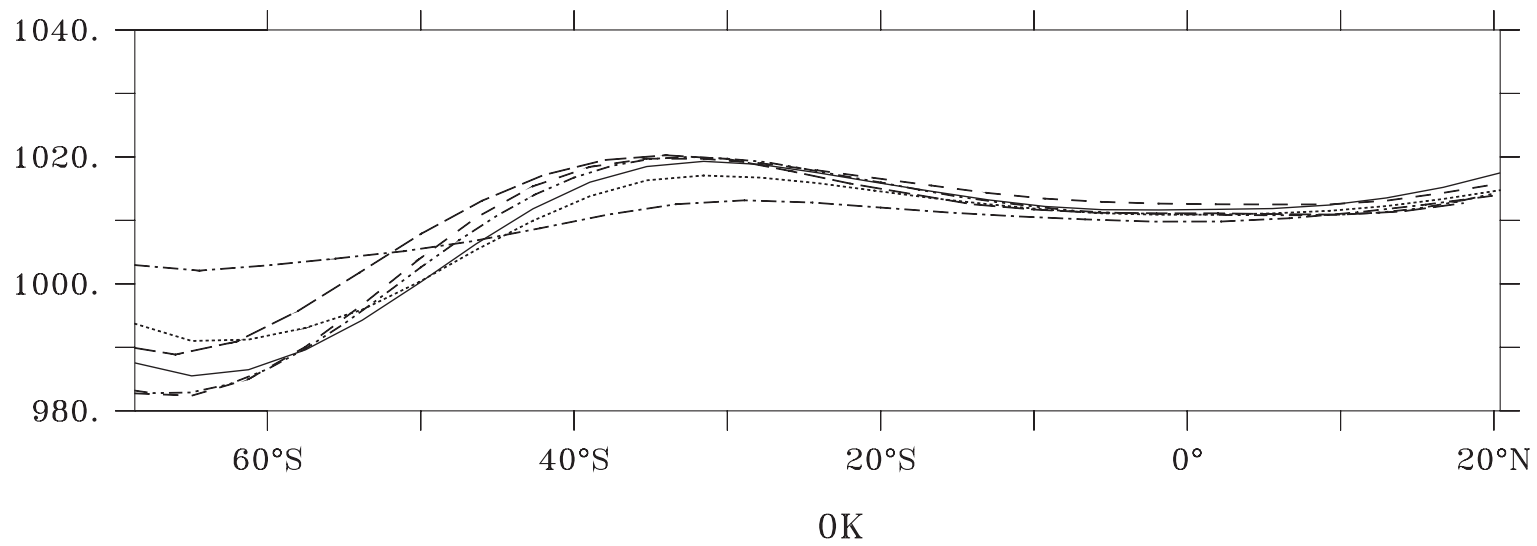

b)

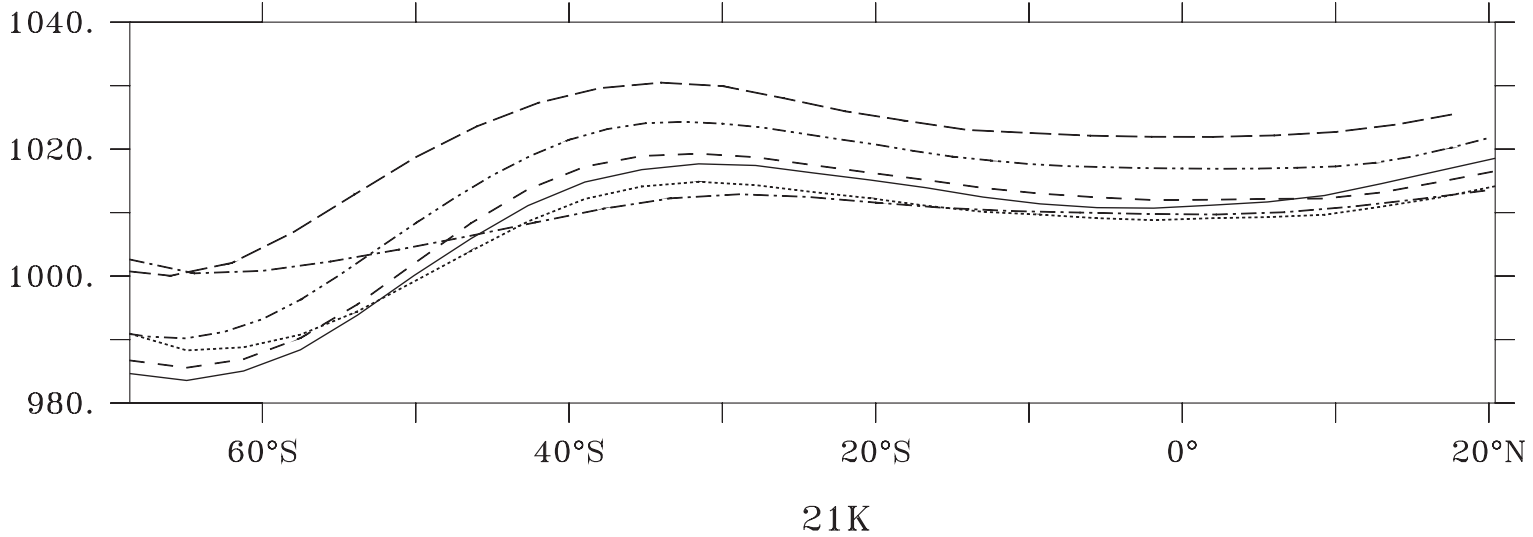

c)

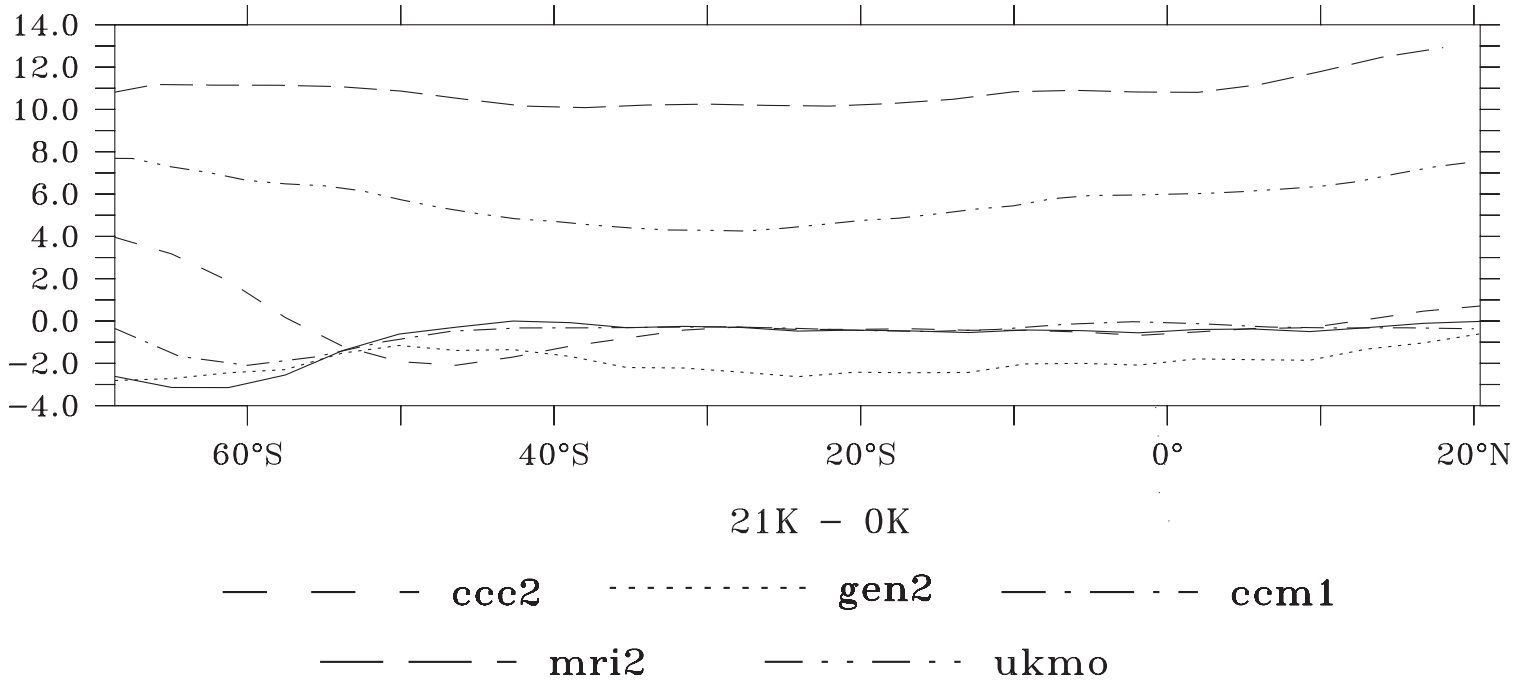

Figura 7 - Média zonal de pressão ao nível do mar para as simulações analisadas no presente (a), no UMG (b) e para a diferença (UMG menos presente) (c). 
Pressao ao Nivel do Mar- 21K-0K

a)

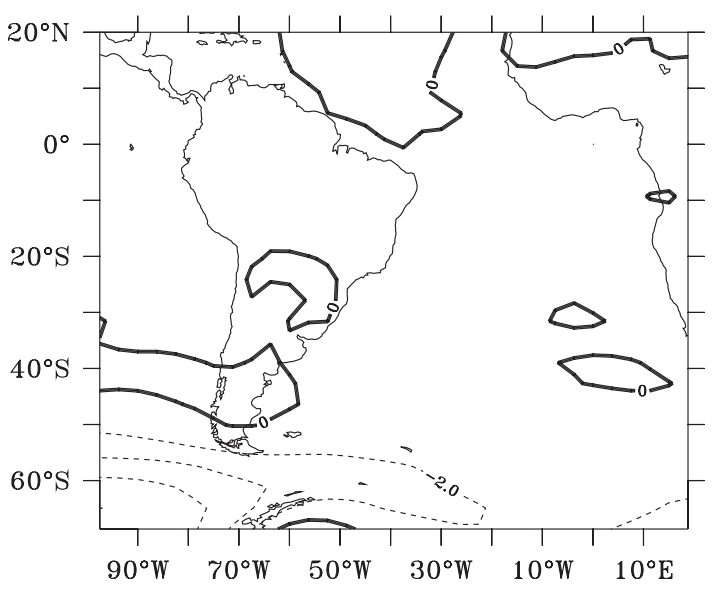

NCAR CCSM

c)

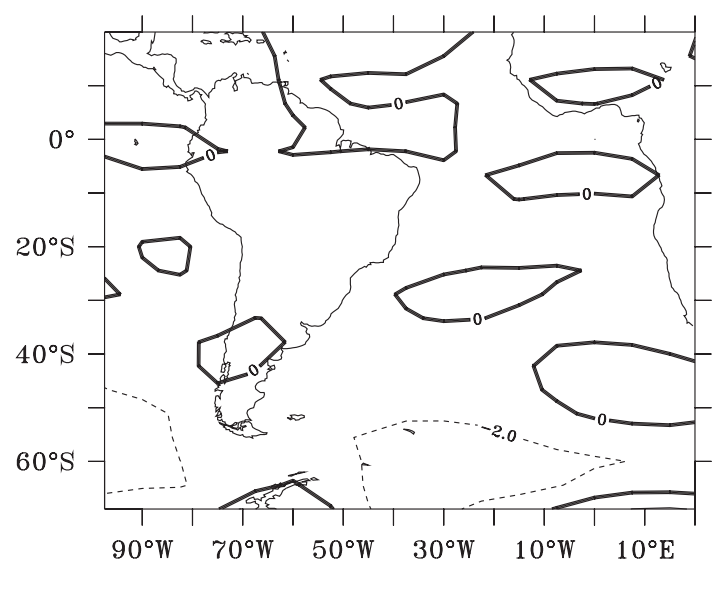

CCM 1

e)

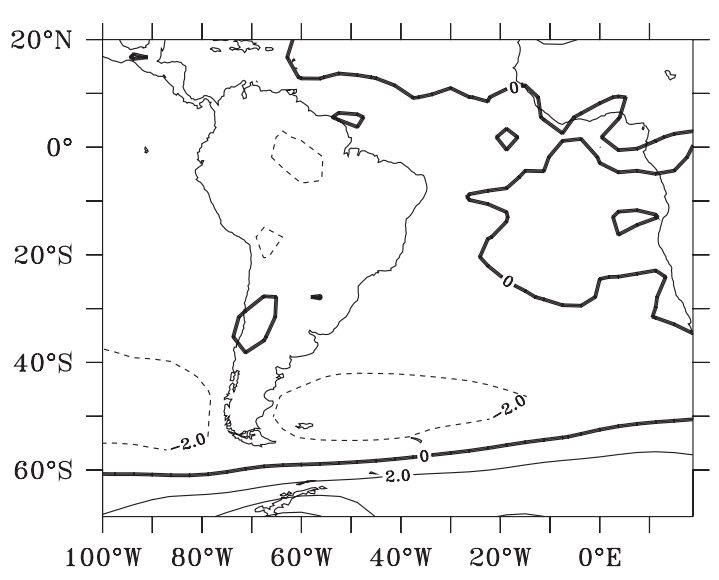

$\mathrm{CCC} 2$ b)

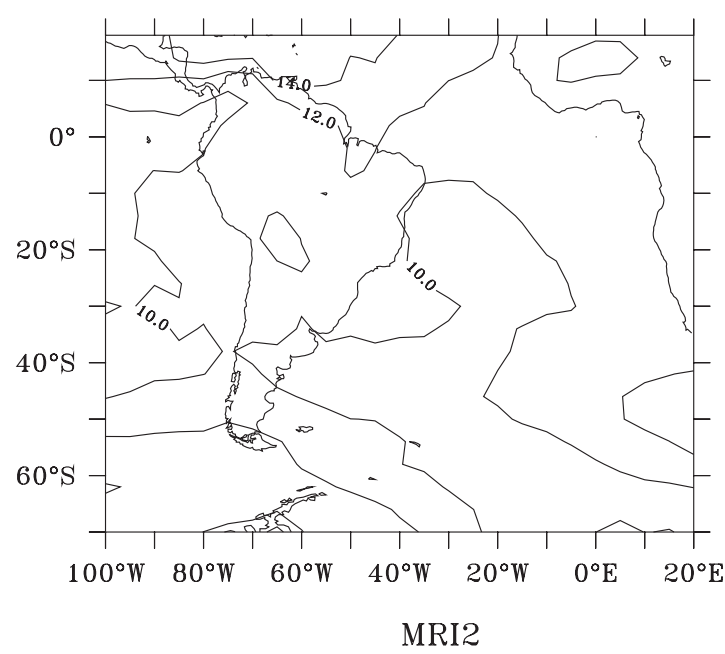

d)

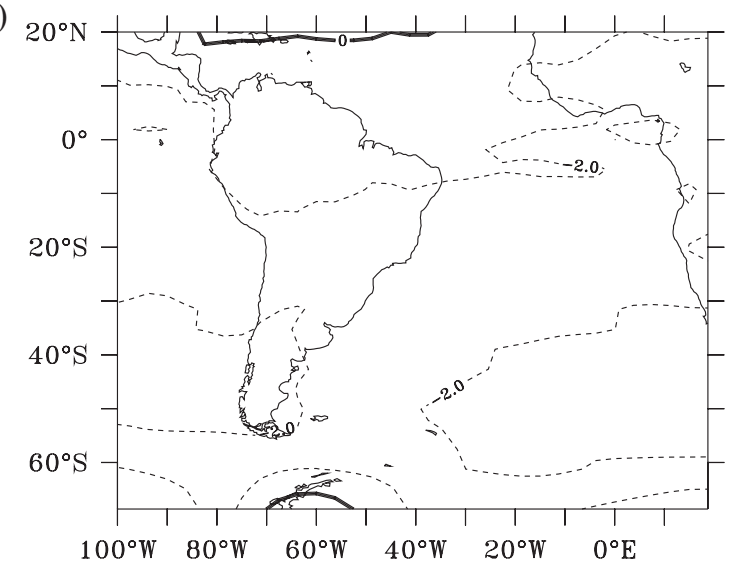

GEN2

f)

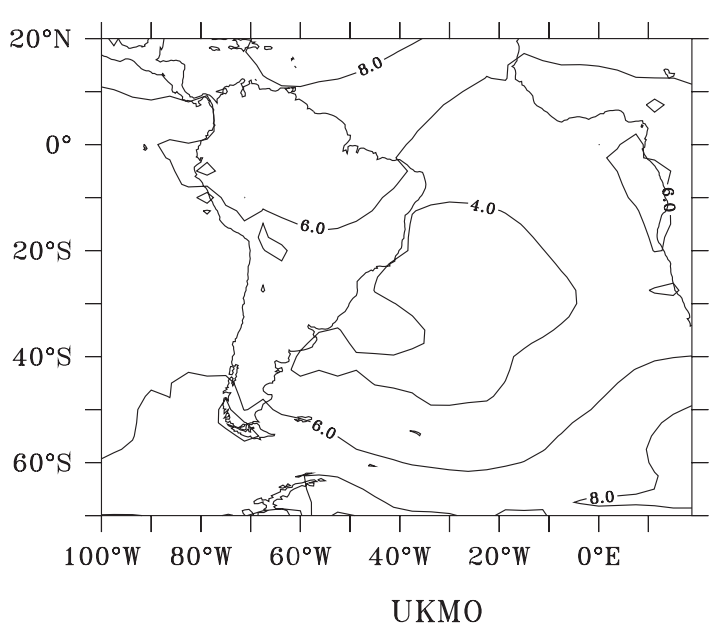

Figura 8 - Diferença das climatologias anuais da pressão ao nível do mar entre os dois períodos estudados dos modelos. Contornos de intervalos de 1mb. (a) NCAR CCSM 1.4, (b) MRI2, (c) CCM1, (d) GEN2, (e) CCC2, (f) UKMO. 


\section{Pressao ao Nivel do Mar - EQM}

a)

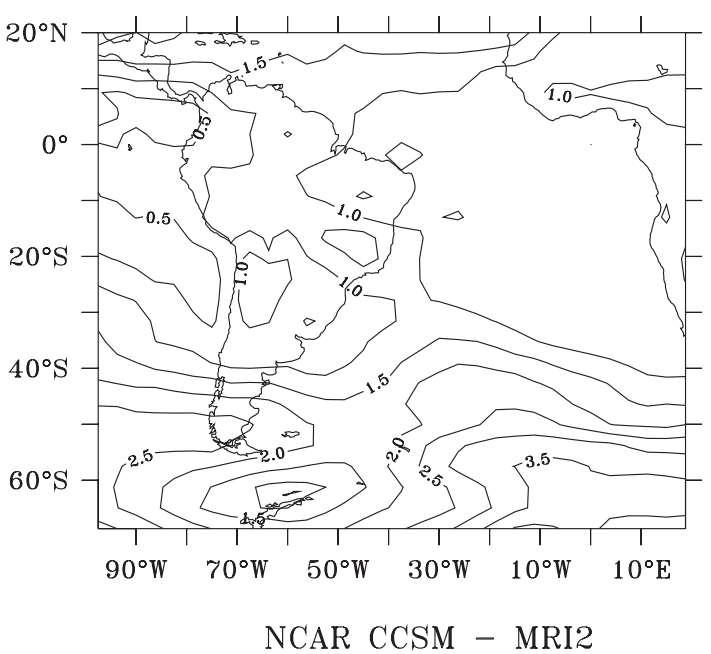

c)

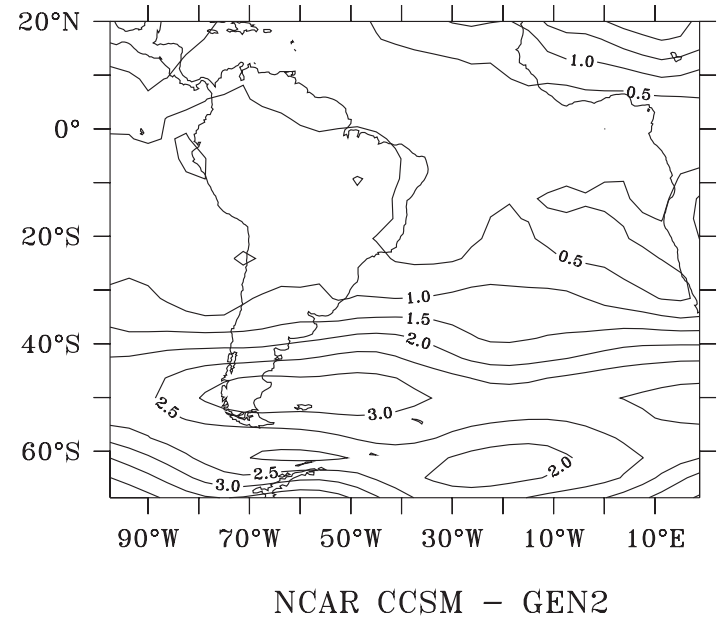

b)

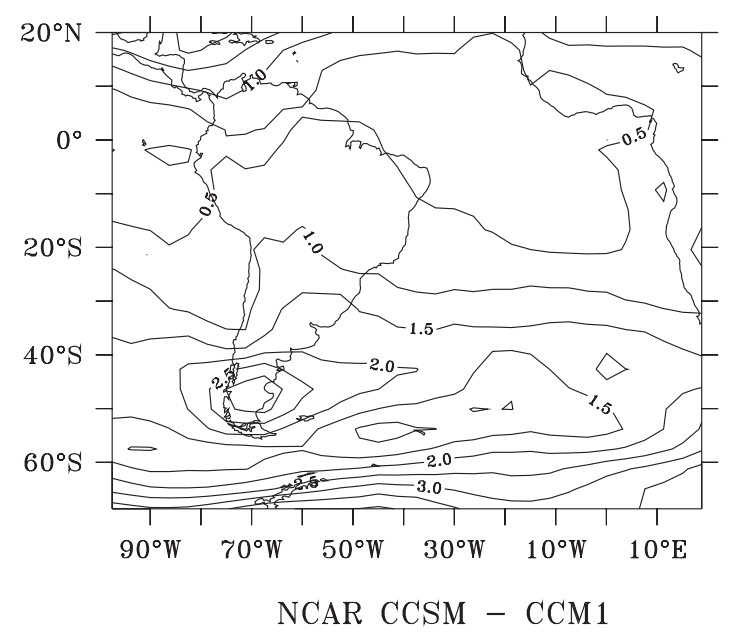

d)

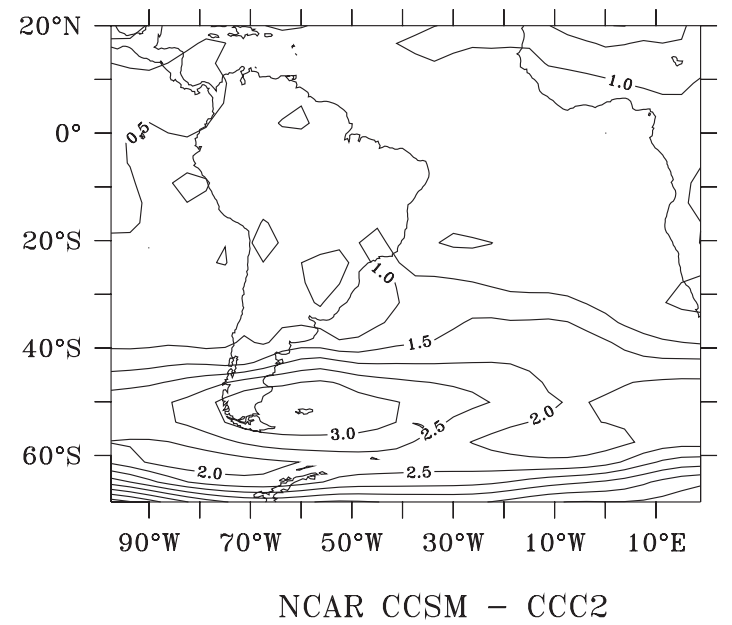

e)

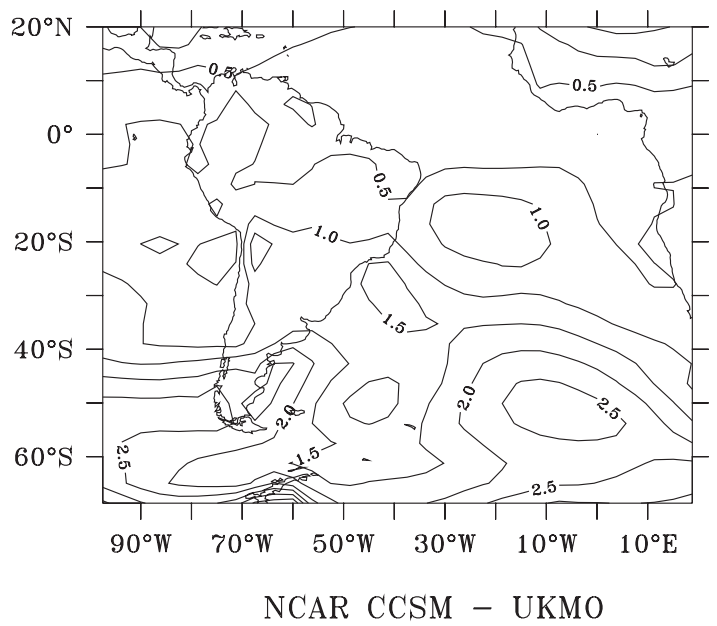

Figura 9 - Análise de EQM para a diferença S entre o modelo NCAR-CCSM 1.4 e os modelos do PMIP. Contornos de intervalos de 0.5mb. (a) NCAR CCSM 1.4 - MRI2, (b) NCAR CCSM 1.4 - CCM1, (c) NCAR CCSM 1.4 - GEN2, (d) NCAR CCSM 1.4 - CCC2, (e) NCAR CCSM 1.4 - UKMO. 


\section{CONCLUSÃO}

Neste trabalho foi realizado um estudo descritivo sobre as mudanças do comportamento climatológico anual de três variáveis em dois períodos distintos: o Último Máximo Glacial e o Presente. Foram analisados os resultados do modelo numérico acoplado oceano-atmosfera NCAR CCSM 1.4 sob as condições atmosféricas dos dois períodos citados anteriormente e 5 simulações do PMIP. Foi analisado e comparado o comportamento das variáveis de temperatura do ar (TA), precipitação (PPT) e pressão atmosférica ao nível do mar (PNM) para todas as simulações. Os resultados mostram de uma maneira geral uma intensificação das variáveis estudadas no presente, com relação ao UMG.

A TA mostrou aquecimento significativo relativo ao período de $21 \mathrm{~K}$ para quase todos os modelos com maior intensidade nos modelos CCM1 e NCAR CCSM, com um aquecimento do presente em relação ao UMG de $12^{\circ} \mathrm{C}$. Espacialmente, esta maior intensidade é observada em altas latitudes, sobre a Corrente Circumpolar Antártica. Quando comparamos o modelo NCAR CCSM 1.4 com os modelos do PMIP podemos observar que as maiores diferenças se encontram nas altas latitudes $\left(\sim 5^{\circ} \mathrm{C}\right)$ local onde o oceano obteve suas maiores variações de temperatura no UMG. A análise de RMS para a TA apresentou baixos valores de EQM $\left(\sim 0.5^{\circ} \mathrm{C}\right)$, mostrando que os campos apresentam um padrão semelhante na maior parte da região de estudo. Estas diferenças podem ser atribuídas a maior variação sazonal observada na extensão da formação de gelo sobre o continente Antártico durante o UMG (Crosta et al., 1998). A representação do gelo Antártico nos modelos ainda é precária.

A climatologia anual de precipitação se mostrou sem muitas mudanças para a maioria dos modelos. O modelo MRI2 apresentou maior variabilidade, com pequenos núcleos de mínimo e máximo de diferença, consistentes com Pinot et al. (1999). De uma maneira geral a precipitação não ultrapassou os $4 \mathrm{~mm} /$ dia para toda região de estudo. Comparado ao modelo acoplado NCAR CCSM 1.4 as simulações do PMIP apresentaram algumas diferenças significativas, podendo novamente destacar o modelo MRI2 que apresentou as maiores diferenças $(\sim 3 \mathrm{~mm} / \mathrm{dia})$. As diferenças entre o modelo MRI2 e o modelo do NCAR se devem ao fato do MRI2 ser um modelo atmosférico simples, possuir uma resolução mais baixa (MRI2 4x5 latxlon) e ter sido rodado por um período mais curto. Observamos para a PPT que os valores de EQM são maiores ( $4 \mathrm{~mm} /$ dia) em baixas latitudes contrariamente ao observado nas outras variáveis. Esta maior variabilidade na região tropical é resultado da dificuldade do modelos numéricos em simular corretamente a complexa dinâmica equatorial, incluindo o ciclo sazonal da ZCIT (Zona de Convergência Inter-tropical) e o regime de chuvas a esta associado na região. Os menores valores de $\operatorname{EQM}(\sim 0.5 \mathrm{~mm} / \mathrm{dia})$ podem ser observados nas demais áreas da região.

Para a PNM a diferença entre um período e outro demonstrou que para alguns modelos (NCAR CCSM 1.4, CCM1, GEN2 e CCC2) a pressão é quase que constante entre os períodos e para outros o UMG apresentou maiores valores de pressão (MRI2 e UKMO). Todos os modelos apresentaram alguma variação na PNM com relação ao modelo acoplado, evidenciando que resultados diferentes podem ser obtidos quando o oceano está acoplado ao modelo atmosférico. A diferença entre os períodos em alguns casos apresentou valores maiores no presente (GEN2 $\sim 2 \mathrm{mb}$ ) e em outros valores menores nesse mesmo período (UKMO e MRI2 $\sim 6 \mathrm{mb}$ ). O campo de EQM para a PNM mostra um padrão espacial semelhante ao da TA. Os menores valores encontrados para a PNM são de aproximadamente $1 \mathrm{mb}$ e os maiores valores são de $4 \mathrm{mb}$.

Os modelos UKMO, MRI2 e CCC2 apresentaram os maiores valores de EQM quando comparados ao NCAR CCSM 1.4 para a TA, PPT e PNM respectivamente. Essas diferenças podem estar relacionadas as diferentes resoluções dos modelos, esquemas de tratamento da superfície terrestre e principalmente do gelo. As condições de contorno também podem estar ser responsáveis pela sensibilidade em altas latitudes. Esses resultados mostram que o uso de somente a componente atmosférica para análise climática pode não ser suficiente para representar de maneira mais completa os processos físicos que controlam o clima. Porém a comparação com dados recontruídos e análises mais complexas é necessária para demonstrar a maior complexidade do modelo acoplado.

\section{AGRADECIMENTOS}

Agradeço a ajuda financeira do $\mathrm{CNPq}$ e do projeto FAPESP n 0 03/09971-7.

\section{BIBLIOGRAFIA}

BARNOLA, J. M.; RAYNAUD, D.; KOROTKEVICH, Y.S. ; LORIUS, C. Vostok ice core provides 160,000-year record of atmospheric $\mathrm{CO}_{2}$. Nature, v. 329, p. 408-414, 1987.

BERGER, A. Reevaluation of the oxygen isotopic composition of planktonic foraminifera: Experimental results and revised paleotemperature equations. Paleoceanography, v. 13, n. 2, p. 150-160, 1978.

BONAN, G. B., The Land Surface Climatology of the NCAR Land Surface Model (LSM 1.0) Coupled to the NCAR Community Climate Model (CCM3). Journal of Climate, v. 11, n. 6,1998 . 
BONFILS, C.; LEWDEN, D.; TAYLOR, K. A summary documentation of the PMIP models. Disponível em $<$ http:// www.pcmdi.lln1.gov/pmip $>$.

BOVILLE, B. A.; GENT, P.R. The NCAR Climate System Model, Version One. Journal of Climate, v. 11, n. 6, 1998.

CLAUZET, Gabriel ; WAINER, Ilana ; LAZAR A ; OTTOBLIESNER, Bette L.; BRADY, Ester C. The South Atlantic Circulation at Last Glacial Maximum using the NCAR Coupled Model. Climate Dynamics, 2004.

CLIMAP (Climate/Long-range Investigation, Mapping and Prediction) Project Members. The surface circulation of ice-age earth. Science,191, 1131-1137, 1976.

CLIMAP, Project Members., Seasonal reconstructions of the Earth's surface at the last glacial maximum. Geological Society of America Map and Chart Series MC-36, 1981.

COOKE, D. W.; HAYS, J.D. Estimates of Antarctic Ocean seasonal sea-ice cover during glacial intervals. In: CRADDOCK, C. Antarctic geoscience (International Union of Geological Sciences, Ser. B, no. 4). Madison, University of Wisconsin Press, p. 1017-1025, 1982.

CROSTA, X.; PITCHON J.J.; BURCKLE, L.H. Reappraisal of Antartic Seasonal Sea-Ice at the Last Glacial Maximum. Geophysics Research Letters, v. 25, n. 14, p. 2703-2706, 1998.

FLATO1, G.M; HIBLER, W.D.III. Modelling Pack Ice as a Cavitating Fluid. Journal Physics Oceanography, v. 22, p. 626-651, 1992.

GATES, W.L. Modelling the ice-age climate. Science, v. 191, 1138-1144, 1976.

GENT, P. R.; BRYAN, F. O.; DANABASOGLU, G.; DONEY, S. C.; HOLLAND, W. R.; LARGE, W. G.; MCWILLIAMS, J. C. The NCAR Climate System Model global ocean component. Journal of Climate, v.11, p. 1287-1306, 1998.

HACK, J. J.; KIEHL, J. T.; HURRELL J. The hydrologic and thermodynamic characteristics of the NCAR CCM3. Journal of Climate, v.11, p.1179-1206, 1998.
HANSEN, J.E.; TAKAHASHI T. Climate Processes and Climate Sensitivity. Geophysic Mono, v. 29, American Geophysic Union, Washington D.C, 1984.

JOUSSAUME, S; TAYLOR, K. E. Status of the Paleoclimate Modeling Intercomparison Project (PMIP). In: Proceedings of the first international AMIP scientific conference. WCRP Report, p. 425-430, 1995.

JOUSSAUME, S.; TAYLOR, K.E. The Paleoclimate Modelling Intercomparison Project, in Paleoclimate Modelling Intercomparison Project (PMIP). In: Proceedings of the Third PMIP workshop-edited by P.Braconnot, p. 25-42, WCRP, La Huardiere, Canada, 4-8 October 1999, 2000.

JOUZEL, J.; RAISBECK, G.; BENOIST, J.-P.; YIOU, F.; LORIUS, C.; RAYNAUD, D.; PETIT, J.-R.; BARKOV, N. I.; KOROTKEVICH, Y. S.; KOTLYAKOV, V. M. A comparison of deep antarctic ice cores and their implications for climate between 65,000 and 15,000 years ago. Quaternary Research, v. 31, p. 135-150, 1989.

KIEHL, J.T., HACK, J.J.; BONAN, G.; BOVILLE, B.A; WILLIAMSON, D.; RASH, P. The National Center fo Atmospheric Research Commynity Climate Model (CCM3). Journal of Climate, v. 11, p. 1131-1149, 1998.

LAUTENSCHLAGER, M.; HERTERICH, K. Atmospheric Response to Ice-Age Conditions - Climatology near the Earth's Surface. Journal Geophysic Research, v. 95, p. 22.547-22.557, 1990

MANABE, S.; HAHN, D.G. Simulation of the tropical climate of an ice age. Journal Geophysic Research, v. 82, p. 5529 5554, 1977.

MILLER, J.R.; RUSSEL, G.L. Ocean heat transport during the last maximum. Paleoceanography, v. 4, p. 141-155, 1989.

OTTO-BLIESNER, B.L; BRADY, E.C. Tropical pacific variability in the NCAR Climate System Model. Journal of Climate, v. 14, p. 3587-3607, 2001.

PELTIER, W.R. In Age Paleotopography. Science, v. 265, p. 195-201, 1994. 
PINOT, S.; RAMSTEIN, G.; HARRISON, S.P.; PRENTICE, I.C.; GUIOT, J.; STUTE, M.; JOUSSAUME, S. Tropical paleoclimates at the Last Glacial Maximum: comparison of Paleoclimate Modeling Intercomparison Project (PMIP) simulation an paleodata. Climate Dynamics, v. 15, p. 857 874, 1999.

RAYNAUD, D.; JOUZEL, J.; BARNOLA, J.; CHAPPELLAZ, J.; DELMAS, R.; LORIUS, C. On the freshwater forcing and transport of the Atlantic thermohaline circulation. Climate Dynamics, v. 12, p. 799-811, 1993.

RIND, D.; PETEET, D. Terrestrial conditions at the last glacial maximum and CLIMAP sea-surface temperature estimates: Are they consistent? Quaternary Research, v. 24, p. 1-22, 1985.

SHIN, S. Understanding the climate of the Last Glacial Maximun using a climate system model.. Tese (Pós-Doutorado) University of Winscosin-Madison, USA, 2002.
SHIN, S.; LIU, Z.; OTTO-BLIESNER, B.; BRADY, E.; KUTZBACH, J.; HARRISON, S. A simulation of the Last Glacial Maximum climate using the NCAR-CCSM. Climate Dynamics, v. 20, p. 127-151, 2003.

THOMPSOM, S.L.; POLLARD, D. Greenland an Antartic mass balance for present and doubled $\mathrm{CO} 2$ from the GENESIS version-2 global climate model. Journal of Climate, v. 10, p. 871-900, 1997.

WEATHERLY, J. W.; BRIEGLEB, B.P.; LARGE, W.G.; MASLANIK, J.A. Sea Ice and Polar Climate in the NCAR CSM. Journal of Climate, v. 11, n. 6, 1998.

WILLIAMS, J.; BARRY, R.G. B; WASHINGTON, W. M. Simulation of the atmospheric circulation using the NCAR global circulation model wiyh ice age boundary conditions. Journal of Apply Meteorology, v. 13, p. 305-317, 1974. 